\title{
In Silico Survey and Characterization of Babesia microti Functional and Non-Functional Proteases
}

\author{
Monica Florin-Christensen ${ }^{1,2, * \mathbb{D}}$, Sarah N. Wieser ${ }^{1,2}$, Carlos E. Suarez ${ }^{3,4} \mathbb{( D}$ and Leonhard Schnittger $1,2 \mathbb{D}$ \\ 1 Instituto de Patobiologia Veterinaria (IPVET), Centro de Investigaciones en Ciencias Veterinarias y \\ Agronomicas, Instituto Nacional de Tecnología Agropecuaria (INTA), Hurlingham C1033AAE, Argentina; \\ wieser.sarah@inta.gob.ar (S.N.W.); schnittger.leonhard@inta.gob.ar (L.S.) \\ 2 Consejo Nacional de Investigaciones Científicas y Técnicas (CONICET), Buenos Aires C1033AAJ, Argentina \\ 3 Animal Disease Research Unit, USDA-ARS, Pullman, WA 99163, USA; suarez@wsu.edu \\ 4 Department of Veterinary Microbiology and Pathology, Washington State University, \\ Pullman, WA 99163, USA \\ * Correspondence: jacobsen.monica@inta.gob.ar
}

\section{check for}

updates

Citation: Florin-Christensen, M.; Wieser, S.N.; Suarez, C.E.; Schnittger, L. In Silico Survey and Characterization of Babesia microti Functional and Non-Functional Proteases. Pathogens 2021, 10, 1457. https: / / doi.org/10.3390/ pathogens 10111457

Academic Editors: Estrella Montero, Jeremy Gray, Cheryl Ann Lobo and Luis Miguel González

Received: 18 September 2021 Accepted: 6 November 2021 Published: 10 November 2021

Publisher's Note: MDPI stays neutral with regard to jurisdictional claims in published maps and institutional affiliations.

Copyright: (c) 2021 by the authors. Licensee MDPI, Basel, Switzerland. This article is an open access article distributed under the terms and conditions of the Creative Commons Attribution (CC BY) license (https:/ / creativecommons.org/licenses/by/ $4.0 /)$.

\begin{abstract}
Human babesiosis caused by the intraerythrocytic apicomplexan Babesia microti is an expanding tick-borne zoonotic disease that may cause severe symptoms and death in elderly or immunocompromised individuals. In light of an increasing resistance of $B$. microti to drugs, there is a lack of therapeutic alternatives. Species-specific proteases are essential for parasite survival and possible chemotherapeutic targets. However, the repertoire of proteases in B. microti remains poorly investigated. Herein, we employed several combined bioinformatics tools and strategies to organize and identify genes encoding for the full repertoire of proteases in the B. microti genome. We identified 64 active proteases and 25 nonactive protease homologs. These proteases can be classified into cysteine $(n=28)$, serine $(n=21)$, threonine $(n=14)$, asparagine $(n=7)$, and metallopeptidases $(n=19)$, which, in turn, are assigned to a total of 38 peptidase families. Comparative studies between the repertoire of B. bovis and B. microti proteases revealed differences among sensu stricto and sensu lato Babesia parasites that reflect their distinct evolutionary history. Overall, this data may help direct future research towards our understanding of the biology and pathogenicity of Babesia parasites and to explore proteases as targets for developing novel therapeutic interventions.
\end{abstract}

Keywords: human babesiosis; Babesia microti; therapeutic drugs; peptidases

\section{Introduction}

Human babesiosis caused by Babesia microti is a malaria-like tick-borne zoonotic disease, first described in the 1950s in the USA, with an increasing number of cases reported ever since in this and other countries around the world [1]. Infections proceed asymptomatic or are accompanied by mild or moderate signs in immunocompetent patients but often lead to severe disease and even death in neonates and the elderly or immunocompromised adults [2].

Some wild rodents act as natural reservoirs of $B$. microti, where the parasite is transmitted both by bites of Ixodes sp. ticks, as well as transplacentally [3,4]. Humans are dead-end hosts and suffer accidental infections mainly through tick bites. Transplacental and blood transfusion-related transmissions have also been documented $[1,5,6]$.

Currently, there is no specific therapy for B. microti human babesiosis [7]. The recommended therapeutic drugs to treat $B$. microt $i$ infections are azithromycin plus atovaquone as the first choice or a combination of clindamycin and quinine as an alternative $[7,8]$. However, the reported appearance of $B$. microti parasites resistant to the first two drugs in chronically infected patients and the negative side effects of the latter two call for the development of alternative therapeutic strategies and increased investments in this field $[2,7-10]$. 
B. microti belongs to the Apicomplexa phylum and, as such, has a mandatory parasitic lifestyle that alternates between its definitive tick host and its intermediate mammalian hosts. Complex physiological processes and molecular interactions between the pathogen and host are needed for invasion, egress, parasite development in the tick stages, and migration processes that lead to the completion of the parasite life cycle and its efficient perpetuation and dissemination. Among the molecules involved in these events, parasite proteases, i.e., enzymes that catalyze proteolytic cleavages, are bound to be of paramount importance $[2,11,12]$.

Indeed, proteases of the model Apicomplexan protozoans Toxoplasma gondii and Plasmodium falciparum have been shown to participate in several essential physiological processes, including nutrient acquisition and processing, invasion and egress from host cells, protein recycling, posttranslational processing, and signal transduction, among others [13-15]. Due to their vital roles and the fact that they show low or no identity with host-encoded peptidases, parasite proteases have been proposed as potential drug targets and/or vaccine candidates [16-20]. In the case of Babesia spp., the importance of peptidases for parasite survival and their potential as therapeutic targets are highlighted by several studies showing that different protease inhibitors significantly impede parasite growth in vitro and/or in vivo [21-25].

The present study aims to shortlist the proteases encoded in the B. microti genome by organizing the information available in the MEROPS protease database, as well as identifying additional peptidases by homology searches for paralogs within the B. microti genome and orthologs of previously described active proteases of B. bovis [26]. We also tested the hypothesis that the repertoires of functional proteases encoded in the genomes of $B$. bovis and B. microti differ, possibly due to the peculiarities displayed in their life cycles and their different phylogenetic placements $[27,28]$. The information recorded in this study can be applied to future research aimed at understanding the biology of this emergent pathogen and designing new therapeutic interventions.

\section{Results and Discussion}

\subsection{Survey of B. microti Proteases}

The present study shows that the B. microti genome encodes for at least 64 active proteases and 25 non active protease homologs. These proteases belong to the cysteine $(n=28)$, serine $(n=21)$, threonine $(n=14)$, aspartic $(n=7)$, and metallopeptidase $(n=19)$ types, which, in turn, are assigned to a total of 38 peptidase families (Table 1 ).

The classification into peptidase types refers to the nature of the nucleophile in the hydrolytic reaction, which can be the thiol of a cysteine in cysteine peptidases, the hydroxyl of a serine, or a threonine residue in serine and threonine peptidases, respectively, or water bound to aspartic acid or to a metal ion in aspartic and metallopeptidases, respectively. An additional protease group has been described, the glutamic peptidases, in which the nucleophile is water bound to a glutamic acid residue, but these enzymes are absent in Apicomplexan protozoa. Peptidases of each type are assigned into families according to sequence similarities. Non active protease homologs are characterized by bearing a conserved protease domain but lacking in the active site one or more of the critical amino acids needed for catalysis [29]. 
Table 1. Proteases belonging to the aspartic, cysteine, threonine, serine, and metallopeptidase types encoded by B. microti.

\begin{tabular}{|c|c|c|c|c|c|c|c|c|}
\hline Type & Family & Protein Id & $\begin{array}{l}\text { GenBank } \\
\text { Annotation }\end{array}$ & Gene Locus & $\begin{array}{l}\text { MEROPS } \\
\text { Annotation }\end{array}$ & $\begin{array}{l}\text { Type and Position of } \\
\text { Peptidase Domain }\end{array}$ & $\begin{array}{c}\text { Active Side } \\
\text { Residues (Active } \\
\text { Proteases) }\end{array}$ & $\begin{array}{l}\text { Transcriptomic/ } \\
\text { Proteomic Data }\end{array}$ \\
\hline \multirow{7}{*}{$\begin{array}{l}\text { Aspartic } \\
\text { proteases }\end{array}$} & \multirow{5}{*}{ A1 } & XP_021337483 & Cathepsin E-B & $\begin{array}{c}\text { BMR1_01G02485 } \\
\text { BBM_I02485 }\end{array}$ & $\begin{array}{c}\text { MER1133958- } \\
\text { subfamily A1A } \\
\text { unassigned } \\
\text { peptidases }\end{array}$ & $\begin{array}{c}\text { PTZ00165 } \\
\text { aspartyl protease } \\
82-403\end{array}$ & $\begin{array}{c}\text { D110, Y158, } \\
\text { D307 }\end{array}$ & $\mathrm{T}$ \\
\hline & & XP_021337801 & Pepsin A & $\begin{array}{c}\text { BmR1_04g07350 } \\
\text { BBM_III07350 }\end{array}$ & $\begin{array}{c}\text { MER0383113 } \\
\text { MER1136315 } \\
\text { subfamily A1A } \\
\text { unassigned } \\
\text { peptidases } \\
\end{array}$ & $\begin{array}{c}\text { cd05471 } \\
\text { pepsin_like } \\
109-417\end{array}$ & $\begin{array}{l}\text { D128, F173, } \\
\text { D324 }\end{array}$ & $\mathrm{T}$ \\
\hline & & XP_021338468 & $\begin{array}{c}\text { Eukaryotic } \\
\text { aspartyl protease }\end{array}$ & $\begin{array}{l}\text { BMR1_03g00915 } \\
\text { BBM_III00915 }\end{array}$ & $\begin{array}{c}\text { MER1142805 } \\
\text { MER0383316 } \\
\text { subfamily A1A } \\
\text { unassigned } \\
\text { peptidases } \\
\end{array}$ & $\begin{array}{c}\text { cd05471 } \\
\text { pepsin_like } \\
133-473\end{array}$ & $\begin{array}{l}\text { D160, F205, } \\
\text { D373 }\end{array}$ & $\mathrm{T}$ \\
\hline & & XP_021338748 & $\begin{array}{c}\text { Eukaryotic } \\
\text { aspartyl protease }\end{array}$ & $\begin{array}{c}\text { BMR1_03g03850 } \\
\text { BBM_III03850 }\end{array}$ & $\begin{array}{c}\text { MER0384385 } \\
\text { subfamily A1A } \\
\text { unassigned } \\
\text { peptidases } \\
\end{array}$ & $\begin{array}{c}\text { PTZ00165 } \\
\text { aspartyl protease } \\
88-401\end{array}$ & $\begin{array}{l}\text { D106, Y160, } \\
\text { D310 }\end{array}$ & $\mathrm{T}$ \\
\hline & & XP_021337625 & Plasmepsin V & $\begin{array}{l}\text { BmR1_04g05270 } \\
\text { BBM_III05270 }\end{array}$ & $\begin{array}{c}\text { MER0495838 } \\
\text { subfamily A1B } \\
\text { unassigned } \\
\text { peptidases }\end{array}$ & $\begin{array}{c}\text { cl11403 } \\
\text { pepsin_retropepsin } \\
\text { like aspartate proteases } \\
167-502\end{array}$ & $\begin{array}{c}\text { D198, Y253, } \\
\text { D388 }\end{array}$ & $\mathrm{T}$ \\
\hline & A22B & XP_021338622 & $\begin{array}{l}\text { Signal peptide } \\
\text { peptidase }\end{array}$ & $\begin{array}{c}\text { BMR1_03g02475 } \\
\text { BBM_III02475 }\end{array}$ & $\begin{array}{c}\text { MER0323102 } \\
\text { subfamily A22B } \\
\text { unassigned } \\
\text { peptidases } \\
\end{array}$ & $\begin{array}{c}\text { cl01342 Peptidase_A22B } \\
\text { Superfamily } \\
27-225\end{array}$ & D115, D156 & $\mathrm{T}$ \\
\hline & A28 & XP_021337501 & $\begin{array}{c}\text { DNA } \\
\text { damage-inducible } \\
\text { protein } 1\end{array}$ & $\begin{array}{l}\text { BMR1_01G02675 } \\
\text { BBM_I02675 }\end{array}$ & $\begin{array}{c}\text { MER0321004 } \\
\text { subfamily A28A } \\
\text { unassigned } \\
\text { peptidases }\end{array}$ & $\begin{array}{c}\text { cd05479 } \\
\text { RP_DDI; } \\
\text { retropepsin-like domain } \\
\text { of DNA damage } \\
\text { inducible protein } \\
\text { 221-342 }\end{array}$ & D231 & $\mathrm{T}$ \\
\hline
\end{tabular}


Table 1. Cont.

\begin{tabular}{|c|c|c|c|c|c|c|c|c|}
\hline Type & Family & Protein Id & $\begin{array}{l}\text { GenBank } \\
\text { Annotation }\end{array}$ & Gene Locus & $\begin{array}{l}\text { MEROPS } \\
\text { Annotation }\end{array}$ & $\begin{array}{l}\text { Type and Position of } \\
\text { Peptidase Domain }\end{array}$ & $\begin{array}{c}\text { Active Side } \\
\text { Residues (Active } \\
\text { Proteases) } \\
\end{array}$ & $\begin{array}{l}\text { Transcriptomic/ } \\
\text { Proteomic Data }\end{array}$ \\
\hline \multirow{6}{*}{$\begin{array}{l}\text { Cysteine } \\
\text { proteases }\end{array}$} & \multirow{5}{*}{$\mathrm{C} 1 \mathrm{~A}$} & XP_021338611 & Cathepsin C & $\begin{array}{l}\text { BMR1_03g02385 } \\
\text { BBM_III02385 }\end{array}$ & $\begin{array}{c}\text { MER0345528 } \\
\text { Subfamily C1A } \\
\text { unassigned } \\
\text { peptidases }\end{array}$ & $\begin{array}{c}\text { PTZ00049 } \\
\text { cathepsin C-like protein } \\
\text { 273-483 }\end{array}$ & $\begin{array}{l}\text { Q274, C280, } \\
\text { H44, D466 }\end{array}$ & $\mathrm{T}$ \\
\hline & & XP_012647584 & $\begin{array}{l}\text { Cysteine } \\
\text { proteinase }\end{array}$ & $\begin{array}{c}\text { BMR1_01G02595 } \\
\text { BBM_I02595 }\end{array}$ & $\begin{array}{l}\text { MER0701894 } \\
\text { Non-peptidase } \\
\text { homolog }\end{array}$ & $\begin{array}{c}\text { PTZ00200 } \\
\text { cysteine proteinase } \\
296-475\end{array}$ & Inactive & $\mathrm{T}$ \\
\hline & & XP_012650559 & $\begin{array}{l}\text { Papain family } \\
\text { cysteine protease }\end{array}$ & $\begin{array}{l}\text { BmR1_04g09925 } \\
\text { BBM_III09925 }\end{array}$ & $\begin{array}{c}\text { MER0344826 } \\
\text { subfamily C1A } \\
\text { unassigned } \\
\text { peptidases }\end{array}$ & $\begin{array}{c}\text { cd02248 Peptidase_C1A } \\
\text { 236-444 }\end{array}$ & $\begin{array}{c}\text { Q252, C258, H38, } \\
\text { N410 }\end{array}$ & $\mathrm{T} / \mathrm{H}$ \\
\hline & & XP_012650562 & $\begin{array}{c}\text { Papain family } \\
\text { cysteine protease }\end{array}$ & $\begin{array}{c}\text { BmR1_04g09940 } \\
\text { BBM_III09940 }\end{array}$ & - & $\begin{array}{l}\text { cd02248 Peptidase_C1A } \\
\text { 236-444 }\end{array}$ & $\begin{array}{l}\text { Q252, C258, } \\
\text { H38, N410 }\end{array}$ & $\mathrm{T}$ \\
\hline & & XP_012647628 & $\begin{array}{l}\text { Papain family } \\
\text { cysteine protease }\end{array}$ & $\begin{array}{c}\text { BMR1_01G02825 } \\
\text { BBM_I02825 }\end{array}$ & $\begin{array}{c}\text { MER0345177 } \\
\text { Unassigned } \\
\text { peptidase } \\
\end{array}$ & $\begin{array}{c}\text { PTZ00200 } \\
\text { cysteine proteinase } \\
324-538 \\
\end{array}$ & $\begin{array}{l}\text { Q342, C348, } \\
\text { H48, N503 }\end{array}$ & $\mathrm{T}$ \\
\hline & $\mathrm{C} 12$ & XP_021337460 & $\begin{array}{c}\text { ubiquitin } \\
\text { carboxyl-terminal } \\
\text { hydrolase L3 }\end{array}$ & $\begin{array}{c}\text { BMR1_01G02185 } \\
\text { BBM_I02185 }\end{array}$ & $\begin{array}{l}\text { MER0342930 } \\
\text { MER1171398 } \\
\text { family C12 } \\
\text { non-peptidase } \\
\text { homologs }\end{array}$ & $\begin{array}{c}\text { cl08306 } \\
\text { Peptidase_C12 } \\
\text { Superfamily } \\
8-224\end{array}$ & Inactive & $\mathrm{T}$ \\
\hline
\end{tabular}


Table 1. Cont.

\begin{tabular}{|c|c|c|c|c|c|c|c|c|}
\hline Type & Family & Protein Id & $\begin{array}{c}\text { GenBank } \\
\text { Annotation }\end{array}$ & Gene Locus & $\begin{array}{l}\text { MEROPS } \\
\text { Annotation }\end{array}$ & $\begin{array}{l}\text { Type and Position of } \\
\text { Peptidase Domain }\end{array}$ & $\begin{array}{c}\text { Active Side } \\
\text { Residues (Active } \\
\text { Proteases) }\end{array}$ & $\begin{array}{l}\text { Transcriptomic/ } \\
\text { Proteomic Data }\end{array}$ \\
\hline & $\mathrm{C} 13$ & XP_012650207 & $\begin{array}{l}\text { GPI-anchored } \\
\text { transamidase }\end{array}$ & $\begin{array}{c}\text { BmR1_04g08080 } \\
\text { BBM_III08080 }\end{array}$ & $\begin{array}{l}\text { MER0674277 glyco- } \\
\text { sylphosphatidyli- } \\
\text { nositol:protein } \\
\text { transamidase }\end{array}$ & $\begin{array}{c}\text { cl00042 } \\
\text { CASc Superfamily } \\
\text { 391-639 }\end{array}$ & H532, C574 & $\mathrm{T}$ \\
\hline & $\mathrm{C} 14$ & XP_012648342 & Caspase domain & $\begin{array}{c}\text { BMR1_02g02900 } \\
\text { BBM_II02900 }\end{array}$ & $\begin{array}{c}\text { MER0393785 } \\
\text { subfamily C14B } \\
\text { unassigned } \\
\text { peptidases }\end{array}$ & $\begin{array}{c}\text { cl00042 } \\
\text { CASc Superfamily } \\
48-168\end{array}$ & Inactive & $\mathrm{T}$ \\
\hline & \multirow{3}{*}{ C19 } & XP_012647713 & $\begin{array}{c}\text { U4/U6.U5 tri- } \\
\text { snRNP-associated } \\
\text { protein } 2\end{array}$ & $\begin{array}{l}\text { BMR1_01G03245 } \\
\text { BBM_I03245 }\end{array}$ & $\begin{array}{l}\text { MER0711213 } \\
\text { family C19 } \\
\text { non-peptidase } \\
\text { homologs }\end{array}$ & $\begin{array}{c}\text { cd02669 } \\
\text { Peptidase_C19M } \\
\text { 158-365 }\end{array}$ & Inactive & $\mathrm{T}$ \\
\hline & & XP_012649658 & $\begin{array}{c}\text { Ubiquitin } \\
\text { carboxyl-terminal } \\
\text { hydrolase } 25\end{array}$ & $\begin{array}{c}\text { BmR1_04g05260 } \\
\text { BBM_III05260 }\end{array}$ & $\begin{array}{l}\text { MER0706972 } \\
\text { family C19 } \\
\text { unassigned } \\
\text { peptidases }\end{array}$ & $\begin{array}{c}\text { cl37989 } \\
\text { UCH Superfamily } \\
839-1135\end{array}$ & $\begin{array}{l}\text { N842, C847, } \\
\text { H1084, D1105 }\end{array}$ & $\mathrm{T}$ \\
\hline & & XP_021338067 & $\begin{array}{c}\text { Ubiquitin } \\
\text { carboxyl-terminal } \\
\text { hydrolase } 5 / 13\end{array}$ & $\begin{array}{l}\text { BMR1_02g00955 } \\
\text { BBM_II00955 }\end{array}$ & $\begin{array}{l}\text { MER0708474 } \\
\text { family C19 } \\
\text { unassigned } \\
\text { peptidases }\end{array}$ & $\begin{array}{c}\text { cl34941 } \\
\text { UBP14 Superfamily } \\
184-673\end{array}$ & $\begin{array}{c}\text { N315, C321, H751, } \\
\text { N746 }\end{array}$ & $\mathrm{T}$ \\
\hline & \multirow[b]{2}{*}{$\mathrm{C} 26$} & XP_012647696 & CTP synthase & $\begin{array}{c}\text { BMR1_01G03155 } \\
\text { BBM_I03155 }\end{array}$ & $1 \quad 1$ & $\begin{array}{c}\mathrm{cl} 33465 \\
\text { CTP synthase } \\
4-570\end{array}$ & C398, H540 & $\mathrm{T}$ \\
\hline & & XP_021337469 & $\begin{array}{c}\text { carbamoyl- } \\
\text { phosphate } \\
\text { synthase// } \\
\text { aspartate car- } \\
\text { bamoyltransferase }\end{array}$ & $\begin{array}{c}\text { BMR1_01G02285 } \\
\text { BBM_I02285 }\end{array}$ & - & $\begin{array}{c}\text { cl36884 } \\
\text { CPSaseII_lrg } \\
\text { Superfamily } \\
459-1571\end{array}$ & C334, H407 & $\mathrm{T}$ \\
\hline
\end{tabular}


Table 1. Cont.

\begin{tabular}{|c|c|c|c|c|c|c|c|c|}
\hline Type & Family & Protein Id & $\begin{array}{l}\text { GenBank } \\
\text { Annotation }\end{array}$ & Gene Locus & $\begin{array}{l}\text { MEROPS } \\
\text { Annotation }\end{array}$ & $\begin{array}{l}\text { Type and Position of } \\
\text { Peptidase Domain }\end{array}$ & $\begin{array}{c}\text { Active Side } \\
\text { Residues (Active } \\
\text { Proteases) } \\
\end{array}$ & $\begin{array}{l}\text { Transcriptomic/ } \\
\text { Proteomic Data }\end{array}$ \\
\hline & $\mathrm{C} 44$ & XP_012650079 & $\begin{array}{l}\text { glucosamine- } \\
\text { fructose-6- } \\
\text { phosphate } \\
\text { aminotransferase }\end{array}$ & $\begin{array}{c}\text { BmR1_04g07400 } \\
\text { BBM_III07400 }\end{array}$ & - & $\begin{array}{c}\text { cl36542 } \\
\text { PTZ00295 super family }\end{array}$ & $\mathrm{C} 40$ & $\mathrm{~T}$ \\
\hline & \multirow[t]{2}{*}{ C48 } & XP_012648199 & $\begin{array}{l}\text { sentrin-specific } \\
\text { protease } 1\end{array}$ & $\begin{array}{l}\text { BMR1_02g02160 } \\
\text { BBM_II02160 }\end{array}$ & $\begin{array}{l}\text { MER0378492 } \\
\text { family C48 } \\
\text { unassigned } \\
\text { peptidases }\end{array}$ & $\begin{array}{c}\mathrm{cl} 23802 \\
\text { Peptidase_C48 } \\
\text { Superfamily } \\
191-358\end{array}$ & $\begin{array}{l}\mathrm{H} 279, \text { D298, Q347, } \\
\text { C353 }\end{array}$ & $\mathrm{T}$ \\
\hline & & XP_021337449 & $\begin{array}{l}\text { sentrin-specific } \\
\text { protease } 2\end{array}$ & $\begin{array}{l}\text { BMR1_01G02005 } \\
\text { BBM_I02005 }\end{array}$ & $\begin{array}{l}\text { MER0378539 } \\
\text { family C48 } \\
\text { unassigned } \\
\text { peptidases }\end{array}$ & $\begin{array}{c}\text { cl23802 } \\
\text { Peptidase_C48 } \\
\text { Superfamily } \\
377-660\end{array}$ & $\begin{array}{l}\text { H453, D583, } \\
\text { Q642, C648 }\end{array}$ & $\mathrm{T}$ \\
\hline & C54 & XP_021337321 & $\begin{array}{l}\text { autophagy-related } \\
\text { protein } 4\end{array}$ & $\begin{array}{l}\text { BMR1_01G00840 } \\
\text { BBM_I00840 }\end{array}$ & 11 & $\begin{array}{c}\text { cl04056 } \\
\text { Peptidase family C54 } \\
\text { 34-256 }\end{array}$ & $\begin{array}{l}\text { Y32, C69, } \\
\text { D218, H220 }\end{array}$ & $\mathrm{T}$ \\
\hline & C56 & XP_012649637 & $\begin{array}{l}\text { 4-methyl-5(b- } \\
\text { hydroxyethyl)- } \\
\text { thiazole } \\
\text { monophosphate } \\
\text { biosynthesis }\end{array}$ & $\begin{array}{l}\text { BmR1_04g05155 } \\
\text { BBM_III05155 }\end{array}$ & $\begin{array}{l}\text { MER0385822 } \\
\text { family C56 } \\
\text { non-peptidase } \\
\text { homologs }\end{array}$ & $\begin{array}{c}\text { cl00020 } \\
\text { GAT_1 superfamily } \\
45^{\prime} 207\end{array}$ & Inactive & $\mathrm{T} / \mathrm{H}$ \\
\hline & $\mathrm{C} 78$ & XP_021337753 & $\begin{array}{l}\text { Peptidase family } \\
\text { C78 }\end{array}$ & $\begin{array}{c}\text { BmR1_04g06690 } \\
\text { BBM_III06690 }\end{array}$ & $\begin{array}{l}\text { MER0393880 } \\
\text { family C78 } \\
\text { unassigned } \\
\text { peptidases }\end{array}$ & $\begin{array}{c}\text { cl06790 } \\
\text { Peptidase family C78 } \\
486-677\end{array}$ & $\begin{array}{c}\text { Y498, C510, D634, } \\
\text { H636 }\end{array}$ & $\mathrm{T}$ \\
\hline & C85A & XP_021337245 & $\begin{array}{l}\text { Ubiquitin } \\
\text { thioesterase otu2 }\end{array}$ & $\begin{array}{l}\text { BMR1_01G00165 } \\
\text { BBM_I00165 }\end{array}$ & $\begin{array}{c}\text { MER0743969 } \\
\text { subfamily C85A } \\
\text { unassigned } \\
\text { peptidases } \\
\end{array}$ & $\begin{array}{c}\text { c19932 OTU Superfamily } \\
\text { OTU-like cysteine } \\
\text { protease } 65-186\end{array}$ & D65, C68, H185 & $\mathrm{T}$ \\
\hline
\end{tabular}


Table 1. Cont.

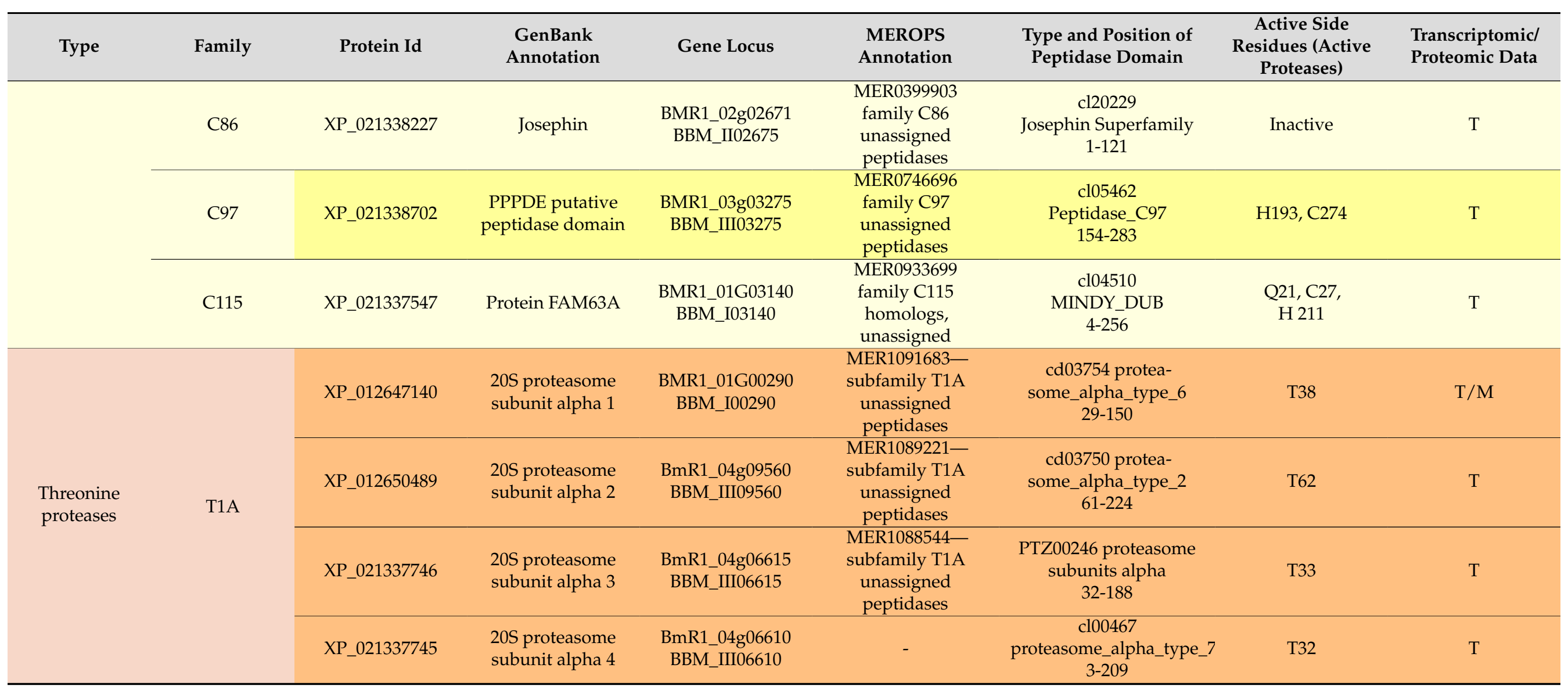


Table 1. Cont.

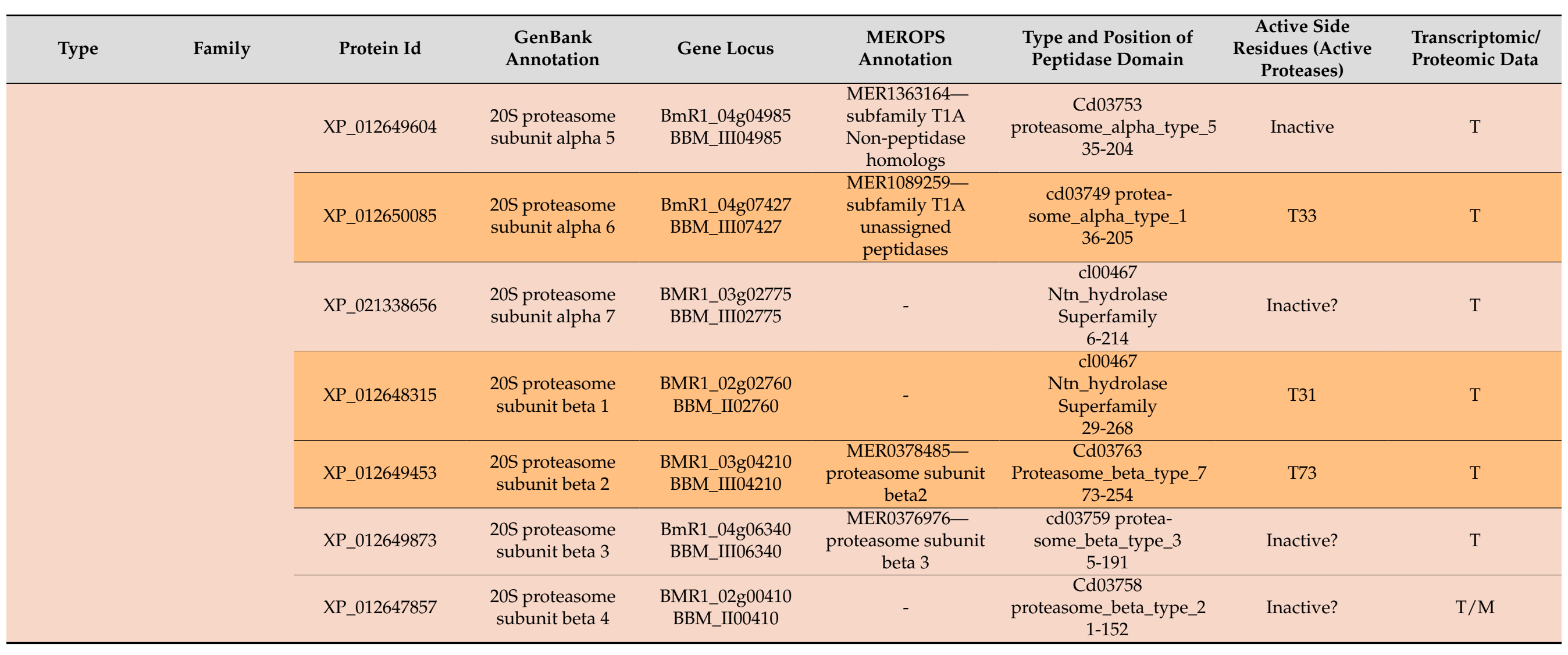


Table 1. Cont.

\begin{tabular}{|c|c|c|c|c|c|c|c|c|}
\hline Type & Family & Protein Id & $\begin{array}{l}\text { GenBank } \\
\text { Annotation }\end{array}$ & Gene Locus & $\begin{array}{l}\text { MEROPS } \\
\text { Annotation }\end{array}$ & $\begin{array}{l}\text { Type and Position of } \\
\text { Peptidase Domain }\end{array}$ & $\begin{array}{c}\text { Active Side } \\
\text { Residues (Active } \\
\text { Proteases) } \\
\end{array}$ & $\begin{array}{l}\text { Transcriptomic/ } \\
\text { Proteomic Data }\end{array}$ \\
\hline & & XP_021338777 & $\begin{array}{l}\text { 20S proteasome } \\
\text { subunit beta } 5\end{array}$ & $\begin{array}{l}\text { BMR1_03g04170 } \\
\text { BBM_III04170 }\end{array}$ & $\begin{array}{l}\text { MER0376387- } \\
\text { subfamily T1A } \\
\text { unassigned } \\
\text { peptidases }\end{array}$ & $\begin{array}{c}\text { cd03761 protea- } \\
\text { some_beta_type_5 } \\
24-226\end{array}$ & $\mathrm{~T} 28$ & $\mathrm{~T}$ \\
\hline & & XP_012650322 & $\begin{array}{l}\text { 20S proteasome } \\
\text { subunit beta } 6\end{array}$ & $\begin{array}{c}\text { BmR1_04g08685 } \\
\text { BBM_III08685 }\end{array}$ & $\begin{array}{l}\text { MER1090686- } \\
\text { subfamily T1A } \\
\text { unassigned } \\
\text { peptidases }\end{array}$ & $\begin{array}{c}\text { cl00467 } \\
\text { Ntn_hydrolase } \\
\text { Superfamily } \\
13-197 \\
\end{array}$ & T13 & $\mathrm{T}$ \\
\hline & & XP_021337419 & $\begin{array}{l}\text { 20S proteasome } \\
\text { subunit beta } 7\end{array}$ & $\begin{array}{l}\text { BMR1_01G01780 } \\
\text { BBM_I01780 }\end{array}$ & $\begin{array}{l}\text { MER1088514- } \\
\text { subfamily T1A } \\
\text { non-peptidase } \\
\text { homologs } \\
\end{array}$ & $\begin{array}{c}\text { cl00467 } \\
\text { Ntn_hydrolase } \\
\text { Superfamily } \\
11-150 \\
\end{array}$ & Inactive & $\mathrm{T}$ \\
\hline \multirow{3}{*}{$\begin{array}{l}\text { Metallo } \\
\text { proteases }\end{array}$} & M01 & XP_012648031 & aminopeptidase $\mathrm{N}$ & $\begin{array}{l}\text { BMR1_02g01305 } \\
\text { BBM_II01305 }\end{array}$ & $\begin{array}{l}\text { MER0335312-M1 } \\
\text { aminopeptidase }\end{array}$ & $\begin{array}{c}\text { PRK14015 pepN } \\
\text { aminopeptidase } \mathrm{N} \\
\text { 366-533 }\end{array}$ & $\begin{array}{c}\text { E423, Y506 metal } \\
\text { ligand(s): H422, } \\
\text { H426, } \\
\text { E445 }\end{array}$ & $\mathrm{T} / \mathrm{M}$ \\
\hline & \multirow[t]{2}{*}{ M16B } & XP_021338255 & $\begin{array}{l}\text { Probable zinc } \\
\text { protease PqqL }\end{array}$ & $\begin{array}{l}\text { BMR1_02g02935 } \\
\text { BBM_II02935 }\end{array}$ & $\begin{array}{l}\text { MER0925233- } \\
\text { subfamily M16A } \\
\text { unassigned } \\
\text { peptidases }\end{array}$ & $\begin{array}{c}\text { COG0612 PqqL } \\
\text { Predicted Zn-dependent } \\
\text { peptidase 14-164 }\end{array}$ & $\begin{array}{c}\text { E40, E116 } \\
\text { metal ligand(s): } \\
\text { H37, H41, E123 }\end{array}$ & $\mathrm{T}$ \\
\hline & & XP_021337876 & $\begin{array}{l}\text { Mitochondrial } \\
\text { processing } \\
\text { peptidase }\end{array}$ & $\begin{array}{l}\text { BmR1_04g08505 } \\
\text { BBM_III08505 }\end{array}$ & $\begin{array}{l}\text { MER0392117- } \\
\text { subfamily M16B } \\
\text { non-peptidase } \\
\text { homologs }\end{array}$ & $\begin{array}{l}\text { COG0612 PqqL } \\
\text { Predicted } \mathrm{Zn} \text {-dependent } \\
\text { peptidase 51-251 }\end{array}$ & Inactive & $\mathrm{T} / \mathrm{H}$ \\
\hline
\end{tabular}


Table 1. Cont.

\begin{tabular}{|c|c|c|c|c|c|c|c|c|}
\hline Type & Family & Protein Id & $\begin{array}{l}\text { GenBank } \\
\text { Annotation }\end{array}$ & Gene Locus & $\begin{array}{l}\text { MEROPS } \\
\text { Annotation }\end{array}$ & $\begin{array}{l}\text { Type and Position of } \\
\text { Peptidase Domain }\end{array}$ & $\begin{array}{c}\text { Active Side } \\
\text { Residues (Active } \\
\text { Proteases) }\end{array}$ & $\begin{array}{l}\text { Transcriptomic/ } \\
\text { Proteomic Data }\end{array}$ \\
\hline & & XP_021338005 & $\begin{array}{l}\text { Mitochondrial } \\
\text { processing } \\
\text { peptidase }\end{array}$ & $\begin{array}{c}\text { BMR1_02g00260 } \\
\text { BBM_IIO0260 }\end{array}$ & $\begin{array}{l}\text { MER0391438- } \\
\text { MER0764852 } \\
\text { mitochondrial } \\
\text { processing } \\
\text { peptidase } \\
\text { beta-subunit }\end{array}$ & $\begin{array}{c}\text { COG0612 PqqL } \\
\text { Predicted Zn-dependent } \\
\text { peptidase } \\
49-253\end{array}$ & $\begin{array}{l}\text { E90, E160 } \\
\text { metal ligand(s): } \\
\text { H87, H91, E167 }\end{array}$ & $\mathrm{T} / \mathrm{H}$ \\
\hline & \multirow[t]{2}{*}{ M16C } & XP_012650528 & $\begin{array}{l}\text { peptidase M16 } \\
\text { inactive domain } \\
\text { containing }\end{array}$ & $\begin{array}{c}\text { BmR1_04g09765 } \\
\text { BBM_III09765 }\end{array}$ & $\begin{array}{l}\text { MER0393094- } \\
\text { subfamily M16C } \\
\text { unassigned } \\
\text { peptidases }\end{array}$ & $\begin{array}{c}\text { PTZ00432 falcilysin } \\
78-550\end{array}$ & $\begin{array}{c}\text { E89, E164 } \\
\text { metal ligand(s): } \\
\text { H86, H90, E203 }\end{array}$ & $\mathrm{T}$ \\
\hline & & XP_021338727 & $\begin{array}{c}\text { Uncharacterized } \\
\text { protein C05D11.1 }\end{array}$ & $\begin{array}{c}\text { BMR1_03g03610 } \\
\text { BBM_III03610 }\end{array}$ & $\begin{array}{l}\text { MER0393111- } \\
\text { subfamily M16C } \\
\text { non-peptidase } \\
\text { homologs }\end{array}$ & $\begin{array}{c}\text { COG1026 Cym1 } \\
\text { Zn-dependent } \\
\text { peptidase, M16 family } \\
54-450\end{array}$ & Inactive & $\mathrm{T}$ \\
\hline & M17 & XP_021338349 & $\begin{array}{c}\text { leucyl } \\
\text { aminopeptidase }\end{array}$ & $\begin{array}{c}\text { BMR1_02g03960 } \\
\text { BBM_II03960 }\end{array}$ & $\begin{array}{l}\text { MER0340008- } \\
\text { family M17 } \\
\text { unassigned } \\
\text { peptidases }\end{array}$ & $\begin{array}{c}\text { PRK00913 } \\
\text { multifunctional } \\
\text { aminopeptidase } \\
\text { a154-499 }\end{array}$ & $\begin{array}{c}\text { K300,R376 metal } \\
\text { ligand(s): A288, } \\
\text { D293, D312, H372, } \\
\text { Q374 }\end{array}$ & $\mathrm{T} / \mathrm{H}$ \\
\hline & M18 & XP_021338536 & aminopeptidase & $\begin{array}{c}\text { BMR1_03g01710 } \\
\text { BBM_III01710 }\end{array}$ & $\begin{array}{l}\text { MER0340957- } \\
\text { MER1122391 } \\
\text { aspartyl } \\
\text { aminopeptidase }\end{array}$ & $\begin{array}{c}\text { cl14876 } \\
\text { Zinc_peptidase_like_Supe } \\
12-481\end{array}$ & $\begin{array}{c}\text { D95, E296 } \\
\text { metal ligand(s): } \\
\text { H93, D255, E297, } \\
\text { D355, H449 }\end{array}$ & $\mathrm{T} / \mathrm{H}$ \\
\hline & M24A & XP_021337644 & $\begin{array}{c}\text { methionyl } \\
\text { aminopeptidase }\end{array}$ & $\begin{array}{c}\text { BmR1_04g05525 } \\
\text { BBM_III05525 }\end{array}$ & - & $\begin{array}{c}\text { PTZ00053 methionine } \\
\text { aminopetidase } 2 \\
9-447\end{array}$ & $\begin{array}{c}\text { H199 } \\
\text { metal ligand(s): } \\
\text { D230, H299, } \\
\text { E332,E427 }\end{array}$ & $\mathrm{T}$ \\
\hline
\end{tabular}


Table 1. Cont.

\begin{tabular}{|c|c|c|c|c|c|c|c|c|}
\hline Type & Family & Protein Id & $\begin{array}{l}\text { GenBank } \\
\text { Annotation }\end{array}$ & Gene Locus & $\begin{array}{l}\text { MEROPS } \\
\text { Annotation }\end{array}$ & $\begin{array}{l}\text { Type and Position of } \\
\text { Peptidase Domain }\end{array}$ & $\begin{array}{c}\text { Active Side } \\
\text { Residues (Active } \\
\text { Proteases) }\end{array}$ & $\begin{array}{l}\text { Transcriptomic/ } \\
\text { Proteomic Data }\end{array}$ \\
\hline & & XP_021337770 & $\begin{array}{c}\text { methionyl } \\
\text { aminopeptidase }\end{array}$ & $\begin{array}{c}\text { BmR1_04g06870 } \\
\text { BBM_III06870 }\end{array}$ & $\begin{array}{c}\text { MER0394794- } \\
\text { methionyl } \\
\text { aminopeptidase 1 }\end{array}$ & $\begin{array}{c}\text { cd1086 MetAP1 } \\
\text { Methionine } \\
\text { Aminopeptidase } \\
70-312\end{array}$ & $\begin{array}{c}\text { H143 } \\
\text { metal ligand(s): } \\
\text { D160, D171, } \\
\text { H234, E267,H298 }\end{array}$ & $\mathrm{T}$ \\
\hline & & XP_021337427 & $\begin{array}{c}\text { methionyl } \\
\text { aminopeptidase }\end{array}$ & $\begin{array}{l}\text { BMR1_01G01855 } \\
\text { BBM_I01855 }\end{array}$ & $\begin{array}{c}\text { MER0395783- } \\
\text { subfamily M24A } \\
\text { unassigned } \\
\text { peptidases }\end{array}$ & $\begin{array}{c}\text { cd1086 MetAP1 } \\
\text { Methionine } \\
\text { Aminopeptidase } \\
\text { 146-514 }\end{array}$ & $\begin{array}{c}\text { H219 } \\
\text { metal ligand(s): } \\
\text { D243, D254, } \\
\text { H423, E455, E486 }\end{array}$ & $\mathrm{T}$ \\
\hline & & XP_012649271 & $\begin{array}{c}\text { methionyl } \\
\text { aminopeptidase }\end{array}$ & $\begin{array}{l}\text { BMR1_03g03300 } \\
\text { BBM_III03300 }\end{array}$ & $\begin{array}{c}\text { MER0394867- } \\
\text { methionyl } \\
\text { aminopeptidase 1 }\end{array}$ & $\begin{array}{l}\text { PLN03158 methionine } \\
\text { aminopeptidase } \\
\text { 105-356 }\end{array}$ & $\begin{array}{c}\text { H179 } \\
\text { metal ligand(s): } \\
\text { D196, D207, } \\
\text { H270, E303, E334 }\end{array}$ & $\mathrm{T}$ \\
\hline & M24B & XP_012650004 & $\begin{array}{c}\text { Xaa-Pro } \\
\text { aminopeptidase }\end{array}$ & $\begin{array}{l}\text { BmR1_04g07005 } \\
\text { BBM_III07005 }\end{array}$ & - & $\begin{array}{c}\text { cd01066 } \\
\text { X-Prolyl } \\
\text { Aminopeptidase }\end{array}$ & $\begin{array}{c}\text { H382,H468,H491 } \\
\text { metal ligand(s): } \\
\text { D401, D412, H472, } \\
\text { E509, E523 }\end{array}$ & $\mathrm{T}$ \\
\hline & \multirow[t]{2}{*}{ M41* } & XP_021338270 & $\begin{array}{l}\text { Peptidase family } \\
\text { M41 }\end{array}$ & $\begin{array}{l}\text { BMR1_02g03060 } \\
\text { BBM_II03060 }\end{array}$ & $\begin{array}{l}\text { MER0363780- } \\
\text { PF14_0616 } \\
\text { g.p. }\end{array}$ & $\begin{array}{c}\text { TIGR01241 FtsH_fam } \\
\text { ATP-dependent } \\
\text { metalloprotease FtsH } \\
\text { 431-647 }\end{array}$ & $\begin{array}{c}\text { E482 } \\
\text { metal ligand(s): } \\
\text { H481, H485, D558 }\end{array}$ & $\mathrm{T} / \mathrm{H}$ \\
\hline & & XP_021338301 & $\begin{array}{l}\text { AFG3 family } \\
\text { protein }\end{array}$ & $\begin{array}{c}\text { BMR1_02g03370 } \\
\text { BBM_II03370 }\end{array}$ & $\begin{array}{c}\text { MER0363828- } \\
\text { family M41 } \\
\text { unassigned } \\
\text { peptidases }\end{array}$ & $\begin{array}{c}\text { TIGR01241 FtsH_fam } \\
\text { ATP-dependent } \\
\text { metalloprotease FtsH } \\
461-682\end{array}$ & $\begin{array}{c}\text { E515 } \\
\text { metal ligand(s): } \\
\text { H514, H518, D581 }\end{array}$ & $\mathrm{T} / \mathrm{H}$ \\
\hline
\end{tabular}


Table 1. Cont.

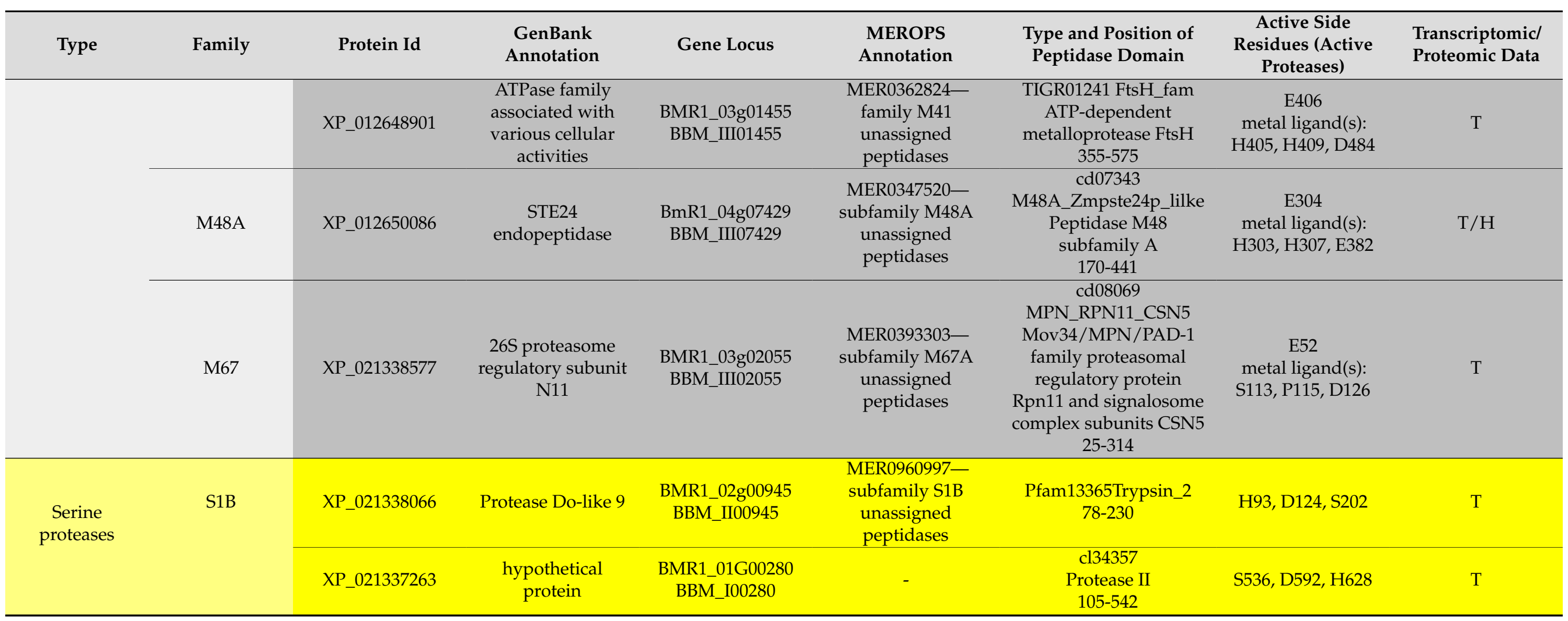


Table 1. Cont.

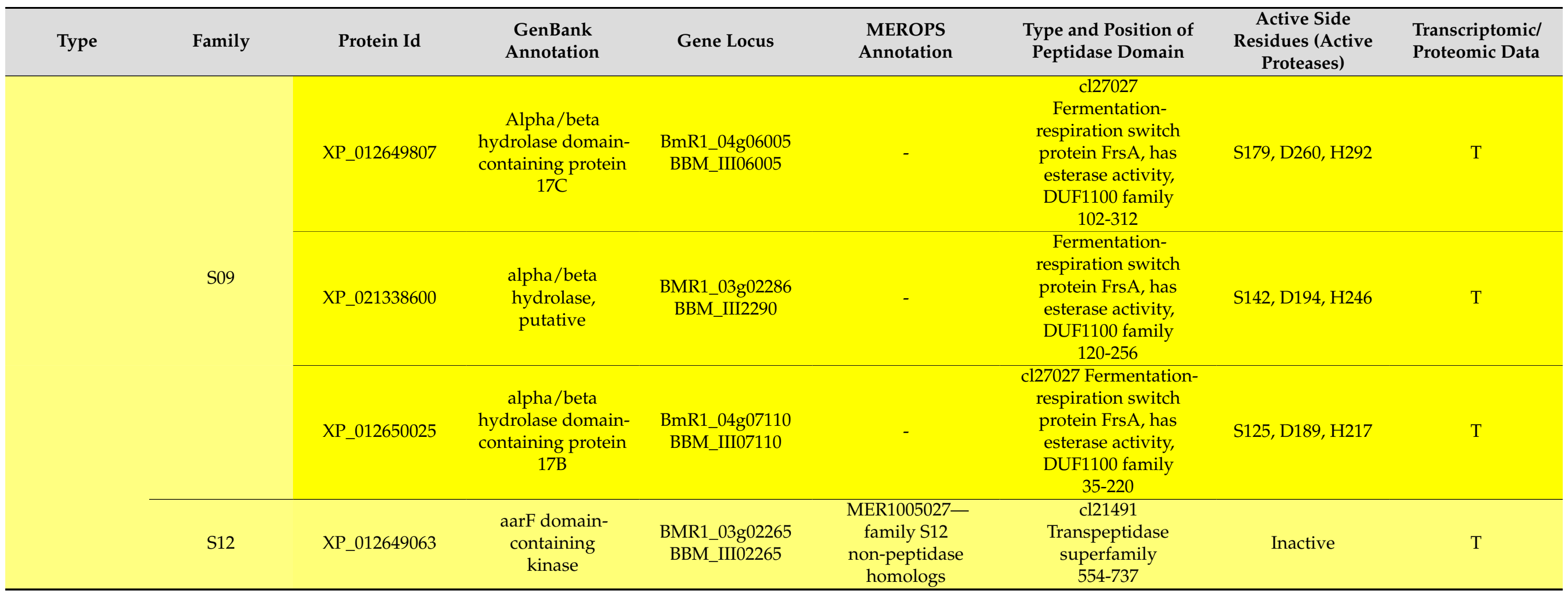


Table 1. Cont.

\begin{tabular}{|c|c|c|c|c|c|c|c|c|}
\hline Type & Family & Protein Id & $\begin{array}{l}\text { GenBank } \\
\text { Annotation }\end{array}$ & Gene Locus & $\begin{array}{l}\text { MEROPS } \\
\text { Annotation }\end{array}$ & $\begin{array}{l}\text { Type and Position of } \\
\text { Peptidase Domain }\end{array}$ & $\begin{array}{c}\text { Active Side } \\
\text { Residues (Active } \\
\text { Proteases) }\end{array}$ & $\begin{array}{l}\text { Transcriptomic/ } \\
\text { Proteomic Data }\end{array}$ \\
\hline & \multirow[t]{2}{*}{ S14 } & XP_012648206 & $\begin{array}{l}\text { ATP-dependent } \\
\text { Clp protease, } \\
\text { protease subunit }\end{array}$ & $\begin{array}{l}\text { BMR1_02g02195 } \\
\text { BBM_IIO2195 }\end{array}$ & $\begin{array}{c}\text { MER0359175- } \\
\text { family S14 } \\
\text { unassigned } \\
\text { peptidases }\end{array}$ & $\begin{array}{c}\text { Cd07017 S14_ClpP_2 } \\
\text { Caseinolytic protease } \\
\text { (ClpP) } \\
41-228\end{array}$ & S135, H160, D209 & $\mathrm{T}$ \\
\hline & & XP_021337686 & Clp protease & $\begin{array}{c}\text { BmR1_04g05887 } \\
\text { BBM_III05890 }\end{array}$ & $\begin{array}{c}\text { MER0359717 } \\
\text { family S14 } \\
\text { non-peptidase } \\
\text { homologs }\end{array}$ & $\begin{array}{c}\text { cd07017 } \\
\text { caseinolytic protease } \\
(\mathrm{ClpP}) \\
50-227\end{array}$ & Inactive & $\mathrm{T}$ \\
\hline & S16 & XP_012649081 & $\begin{array}{l}\text { Lon protease } \\
\text { homolog } 1 \\
\text { mitochondrial }\end{array}$ & $\begin{array}{l}\text { BMR1_03g02350 } \\
\text { BBM_III02350 }\end{array}$ & $\begin{array}{c}\text { MER0361396- } \\
\text { family S16 } \\
\text { unassigned } \\
\text { peptidases } \\
\end{array}$ & $\begin{array}{c}\mathrm{cl} 36736 \\
\text { Ion endopeptidase La } \\
\text { 835-1038 }\end{array}$ & S946, K989 & $\mathrm{T} / \mathrm{H}$ \\
\hline & \multirow[t]{2}{*}{ S26 } & XP_021338290 & $\begin{array}{c}\text { mitochondrial } \\
\text { inner membrane } \\
\text { protease subunit } 1\end{array}$ & $\begin{array}{l}\text { BMR1_02g03240 } \\
\text { BBM_II03240 }\end{array}$ & $\begin{array}{c}\text { MER1047726- } \\
\text { subfamily S26A } \\
\text { non-peptidase } \\
\text { homologs }\end{array}$ & $\begin{array}{c}\text { Cd06530 S26_SPase_I } \\
64-102\end{array}$ & Inactive & $\mathrm{T}$ \\
\hline & & XP_012650493 & $\begin{array}{l}\text { signal peptidase, } \\
\text { endoplasmic } \\
\text { reticulum-type }\end{array}$ & $\begin{array}{c}\text { BmR1_04g09580 } \\
\text { BBM_III09580 }\end{array}$ & $\begin{array}{c}\text { MER0334095- } \\
\text { signalase (animal) } \\
21 \text { kDa component }\end{array}$ & $\begin{array}{c}\text { cl10465 } \\
\text { Peptidase_S24_S26 } \\
\text { Superfamily } \\
52-169\end{array}$ & S63, H101 & $\mathrm{T} / \mathrm{M}$ \\
\hline & S33 & XP_012648716 & $\begin{array}{l}\text { cardiolipin- } \\
\text { specific } \\
\text { phospholipase }\end{array}$ & $\begin{array}{l}\text { BMR1_03g00525 } \\
\text { BBM_III00525 }\end{array}$ & - & $\begin{array}{c}\text { cl21494 Abhydrolase_1 } \\
\text { 98-327 }\end{array}$ & S172, D292, H350 & $\mathrm{T}$ \\
\hline & \multirow[t]{2}{*}{ S54 } & XP_021338360 & $\begin{array}{l}\text { Rhomboid-like } \\
\text { protease } 6\end{array}$ & $\begin{array}{l}\text { BMR1_02g04085 } \\
\text { BBM_II04085 }\end{array}$ & $\begin{array}{c}\text { MER1084044- } \\
\text { family S54 } \\
\text { unassigned } \\
\text { peptidases }\end{array}$ & $\begin{array}{c}\mathrm{cl} 21536 \\
\text { Rhomboid Superfamily } \\
\text { 363-474 }\end{array}$ & S391, H452 & $\mathrm{T}$ \\
\hline & & XP_021338239 & ROM4 & $\begin{array}{l}\text { BMR1_02g02777 } \\
\text { BBM_II02780 }\end{array}$ & $\begin{array}{l}\text { MER0374041- } \\
\text { family S54 } \\
\text { unassigned } \\
\text { peptidases } \\
\end{array}$ & $\begin{array}{c}\text { cl21536 } \\
\text { Rhomboid Superfamily } \\
177-339\end{array}$ & S270, H322 & $\mathrm{T}$ \\
\hline
\end{tabular}


Table 1. Cont.

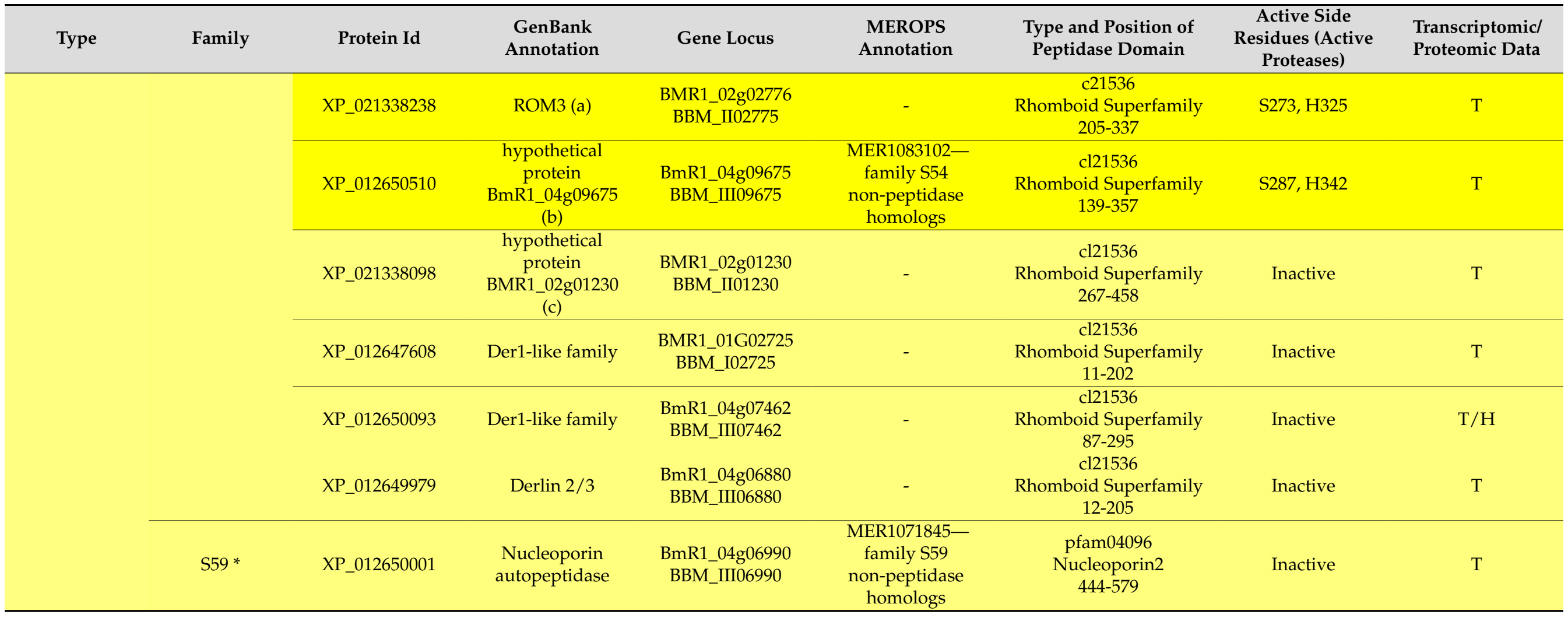

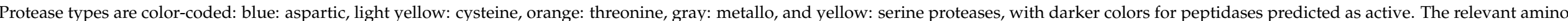

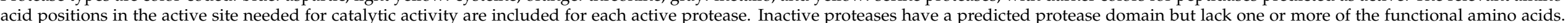

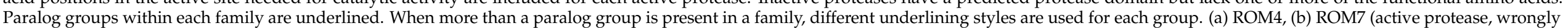

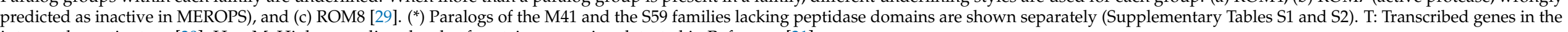
intraerythrocytic stage [30]. H or M: High or medium levels of protein expression detected in Reference [31] 
Twenty of the proteases presented in this study are not included in the MEROPS database and were identified by homology searches. In addition, five of the proteases listed in Table 1 presented duplicated MEROPS entries, likely because of the use of different sources of peptide sequences in this database (Table 1). On the other hand, a number of $B$. microti proteases have been annotated in GenBank as hypothetical proteins, uncharacterized proteins, or following the designation of another conserved domain also present in the sequences, and they are, thus, not identifiable by searches using keywords such as protease or peptidase. Importantly, despite the exhaustive search carried out to produce the list presented in Table 1, the presence of additional protease-coding genes in the B. microti genome that passed inadvertently in this study cannot be ruled out. In addition, it should be noted that the predicted catalytic activity for some threonine proteases (Table 1) could not be determined beyond doubt and needs to be confirmed experimentally.

All of the listed active and non active proteases are transcribed in B. microti merozoites, suggesting they likely fulfill a relevant functional role in this parasite stage [30]. In addition, 17 active and non active proteases were identified in the proteomic profile of $B$. microti during the acute infection of a hamster model (Table 1) [31]. Proteases that remained undetected might be expressed in low amounts at the intraerythrocytic stage or bear physicochemical characteristics that preclude detection by the experimental approach employed in this study [31].

Localization predictor algorithms located most of the identified proteases in the cytoplasm. Four proteases were predicted as extracellular and six as lysosomal. The latter might reach the extracellular milieu by the fusion of vacuoles with the plasma membrane, as has been shown for Tetrahymena thermophila, a free-living protozoon belonging together with Apicomplexa to Alveolata. However, this mechanism has not been demonstrated for Babesia spp. [32]. Other predicted locations include the nucleus, the mitochondria, a plastid (which would correspond to the apicoplast, in this case), the plasma membrane, the endoplasmic reticulum, the Golgi apparatus, and the peroxisomes (Supplementary Table S3). Importantly, these predictions are only tentative until they have been experimentally confirmed. Additionally, the used algorithm is not able to predict the location of proteases in Apicomplexa-specific secretory organelles, such as rhoptries and micronemes, where the trafficking of proteases has been shown to occur in Plasmodium and Toxoplasma [33].

\subsection{Aspartic Proteases}

Seven aspartic proteases were found in the B. microti-predicted proteome, all of which bear the aspartate and, in the case of the A1 family, also the phenylalanine or tyrosine residues in their active sites, needed to display catalytic activity (Table 1).

Interestingly, a recent transcriptomics study involving four of the five A1 aspartic protease genes of $B$. microti showed stage-associated expression for two of them. Thus, while BmR1_01G02485 (encoding cathepsin E-B or ASP2) displayed higher expression in mouse blood intraerythrocytic stages than in the stages present in I. ricinus gut or salivary glands, the opposite was true for BmR1_03g03850 (encoding ASP6). These results suggest a role for ASP2 in processes connected to the asexual reproduction of the parasite and/or gametocyte formation and, for ASP6, in zygote and/or kinete development, kinete dissemination in tick tissues, including salivary glands, and sporogony. For the other two studied A1 aspartic protease-encoding genes, BmR1_04g07350 and BmR1_04g05270, corresponding to ASP3 and ASP5, respectively, expression was similar in the three stages, suggesting a role in invasion both of erythrocytes and tick cells or in other cellular processes such as secretion or the trafficking of proteins [34]. 
Aspartic proteases have been proposed as chemotherapeutic targets against B. microti. Indeed, the aspartic protease inhibitors Lopinavir and Atazanavir, which are well-tolerated drugs used in HIV patients, were shown to be potent suppressors of $B$. microti infection in vitro, as well as in vivo, in a mouse model [24]. It is unknown which parasite aspartic protease is affected by these inhibitors, but one candidate is the signal peptide peptidase (SPP, XP_021338622), which has a critical role in the maintenance of the homeostasis of the endoplasmic reticulum. Consistent with this view, in the case of $P$. falciparum, these inhibitors were effective in blocking SPP activity and in vitro parasite growth [24,35]. Notably, B. microti and Plasmodium sp. SPP proteins are orthologous (results not shown) but do not have a counterpart in B. bovis (Supplementary Table S4) or any other Babesia sp. (not shown).

Hemoglobin is certainly the main protein source available for the nutrition of intraerythrocytic parasites. The sequential steps for hemoglobin degradation by Plasmodium sp., as described by Guzman et al. 1994 [36], start with the unwinding of the molecule and partial digestion by aspartic proteases, followed by cysteine protease cleavage, which yields protein fragments that are finally degraded by exopeptidases, generating free amino acids useful for parasite nutrition. In Plasmodium sp., the first part of this process takes place in the food vacuole and involves the aspartic proteases Plasmepsins I-IV and is then followed by the action of papain-like cysteine proteases in the erythrocyte cytoplasm [37]. There is no evidence that a food vacuole is present in B. microti, and consistent with its absence, Plasmepsins I-IV homologs cannot be found in this parasite. The lack of these enzymes has been used as an argument to postulate that B. microt $i$ is not able to degrade hemoglobin [38]. However, it may be hypothesized that other B. microti aspartyl proteases of the A1 family (Table 1), likely secreted to the erythrocyte cytoplasm, are able to initiate hemoglobin degradation, such as pepsin A (XP_021337801), predicted to have a signal peptide and, thus, be exported to the erythrocyte cytoplasm (Supplementary Table S3). To find out whether this is the case or there is an alternative protein source available for the nutrition of the intraerythrocytic trophozoite and merozoite stages would need experimental exploration.

\subsection{Cysteine Proteases}

Cysteine proteinases are involved in the essential biological roles of Apicomplexan parasites $[13,39,40]$. They are present in $B$. microt $i$ with at least 27 members, of which 18 are predicted to be catalytically active (Table 1 ).

In $P$. falciparum, the papain-like falcipain- 2 and falcipain-3 peptidases of the C1A family have attracted the most attention among cysteine proteases as potential therapeutic targets against malaria [41]. As mentioned above, these enzymes participate in hemoglobin degradation in the intraerythrocytic stage of the parasite, and, in addition, falcipain-2 has been shown to cleave erythrocyte cytoskeletal proteins during egress from the host cell $[42,43]$. Falcipain-2 orthologs have been characterized in B. bovis, B. bigemina, and B. ovis and have been named bovipain-2, babesipain, and ovipain-2, respectively. Similar to their $P$. falciparum counterpart, they are expressed inside merozoites and also released to the erythrocyte cytoplasm, consistent with the dual role described for falcipain-2 [44-47]. The significant impairment of the in vitro growth of B. ovis and B. bovis merozoites by antibodies against ovipain-2 and a papain-like C1A cysteine protease, respectively, indicate a relevant role of this type of enzymes in the propagation of the asexual stages of Babesia spp. $[47,48]$. 
The B. microti ortholog of falcipain-2 (XP_012650559) has four paralogs (three active proteases and one non-protease homolog), one of which is 100\% identical (XP_012650562; Table 1). The corresponding genes for these two identical proteins are located on the same strand of chromosome 3, separated by a $\sim 5 \mathrm{~kb}$ intergenic region, where two unrelated genes are found in the opposite strand. Predictor algorithms localized XP_012650559 and XP_012650562, either within lysosomes or other vacuoles or secreted through a non classical pathway (Supplementary Table S3). This predicted localization agrees well with that described for their counterparts in B. bovis, B. bigemina, and B. ovis [44-48]. In a recent study, an enzymatically active recombinant form of B. microti XP_012650559 (rBmCYP) was expressed in E. coli. The activity of $\mathrm{rBmCYP}$ against a fluorescent peptide was significantly inhibited by recombinant forms of the cysteine protease inhibitors cystatins 1 and 2 of Riphicephalus haemaphysaloides ticks [49]. Although $R$. haemaphysaloides is not a typical $B$. microti-transmitting tick, it has been suggested as a potential vector for this parasite in China [50]. These results coincide with the inhibition exerted by $R$. microplus cystatins on a $B$. bovis $\mathrm{C} 1 \mathrm{~A}$ cysteine protease and suggest the involvement of these enzymes in tick host-pathogen interactions [51]

Interestingly, the phylogenetic analysis of C1A cysteine protease paralog profiles of piroplasmids of the Babesia, Theileria and Cytauxzoon genera corroborates the assignment of analyzed species into Clades I-VI according to their $18 \mathrm{~S}$ rRNA gene sequences [27,52].

\subsection{Serine Proteases}

At least thirteen functional serine proteases and eight non functional protease homologs belonging to nine families are encoded in the B. microti genome (Table 1 ).

A prominent group of serine proteases is constituted by the S54 family, which consists of rhomboid proteases (ROMs). ROMs were first described in Drosophila melanogaster and later shown to be present in all kingdoms of life, fulfilling various relevant roles, including cell signaling in animals, quorum sensing in bacteria, homeostasis regulation in mitochondria, and the dismantling of adhesion complexes in apicomplexan protozoa. They are characterized by having six to seven transmembrane domains and their active site embedded in the lipid bilayer [53,54].

ROMs have been thoroughly studied in the apicomplexans Toxoplasma gondii and Plasmodium spp. The former encodes ROM1-6, according to the nomenclature defined by Dowse and Soldati, 2005 [55], all of which have, with exception of ROM2, homologs in $P$. falciparum. The latter parasite has four additional ROMs that are not present in T. gondii, designated ROM7-10 [56,57]. T. gondii and Plasmodium sp. ROM4 proteases were shown to cleave parasite adhesins, thus dismantling the adhesive junctions formed between the membranes of the host and parasite, a process needed for parasite internalization into the host cell [53,57]. Due to their critical role in invasion, ROMs are regarded as potential targets for therapeutic interventions against apicomplexans [58]. Indeed, two ROM4 inhibitors were shown to specifically block the P. falciparum invasion of human erythrocytes [59]. Additionally, experimental vaccine formulations based on T. gondii and Emeria tenella ROM4 were able to partially protect mice and chickens, respectively, against challenges $[20,60]$.

In a recent study, ROM-coding genes were identified in the genomes of several piroplasmids and shown to belong exclusively to the ROM4, ROM6, ROM7, and ROM8 types. While the latter three were always present in a single copy, two to five ROM4 paralogs could be found depending on the piroplasmid lineage analyzed [61]. B. microti has two ROM4 paralogs, one of which (XP_021338238) has been misannotated as "ROM3" in GenBank (Table 1). ROM4 proteinases are found exclusively throughout the phylum Apicomplexa, which is consistent with their predicted role in invasion of the host cell, a critical mechanism for these obligate parasites [56]. ROM6, on the other hand, is the only piroplasmid rhomboid not exclusive to apicomplexans and has been shown to participate in various processes, including mitochondrial homeostasis, apoptosis, and the electron transport chain [62]. Accordingly, a mitochondrial localization was predicted for B. microti ROM6 (XP_021338360; Supplementary Table S3). B. microti ROM7 (XP_012650510) and ROM8 
(XP_021338098) were predicted to localize in the membranes of the endoplasmic reticulum and the Golgi apparatus, respectively (Supplementary Table S3). These two types of ROMs are present in Plasmodium sp. and piroplasmids but not in other apicomplexans. Their functions are unknown but could be related to processes shared by all Aconoidasida, such as those that take place during the intraerythrocytic stage [61]. Finally, three members of the "derlin" subfamily were found in B. microti (Table 1). Derlins are catalytically inactive members of the Rhomboid Superfamily and were first described in yeast and later found in mammals and other organisms. Their function is still unclear, but it has been suggested that they could be part of a channel through which misfolded proteins are retrotranslocated from the endoplasmic reticulum to the cytoplasm prior to their ubiquitination and degradation [63].

Notably, for B. bovis, one of the ROM-encoding genes (XP_001610128) was found to be significantly higher expressed in the parasite stages present in the hemolymph of Rhipicephalus microplus ticks as compared to the stages present in bovine blood, suggesting that the role of this protease is mostly associated with the development of the parasite in the tick [64]. It remains to be analyzed whether a similar scenario takes place for the corresponding orthologs in B. microti and other piroplasmids.

In an early study, the serine protease activity of $B$. bovis merozoite homogenates was found to be higher in two virulent than in two avirulent strains from Australia, and thus, these proteases were postulated as virulence determinants [65]. However, in a later study, all the genes encoding for active proteases $(n=66)$ were shown to be present and transcribed to similar levels in the asexual blood stages of a B. bovis virulent parental strain and an attenuated strain, obtained by successive blood passages in splenectomized bovines [26]. These data suggest that the virulent/attenuated phenotype in this parasite is not related to a different peptidase gene content or to changes in the transcriptional levels of any peptidase-coding gene. To establish whether or not parasite serine or other types of proteases are virulence determinants in Babesia spp. will need further experimental evidence, but in any case, their relevance for pathogenicity is based on the vital role they probably fulfill in the parasitic lifestyle.

\subsection{Metalloproteases}

Metalloproteases contain a metal ion at their active site, which acts as a catalyst in the hydrolysis of peptide bonds, and are represented by at least 17 active and two non active protease homologs in B. microti (Table 1) [29].

Among them, methionine aminopeptidases (MAPs), which are present with four members in B. microti (M24A family, Table 1), take care of the N-terminal methionine excision from polypeptides, general metabolism of amino acids and proteins, and regulation processes that imply the activation and inactivation of biologically active peptides [66]. Inhibitors of MAPs significantly reduced the in vitro growth of $P$. falciparum, B. bovis, $B$. bigemina, B. caballi, and T. equi, highlighting a relevant role of MAPs in the survival of these parasites [23,67]. Moreover, $B$. microti-infected mice treated with MAP inhibitors reached significantly lower parasitemia levels than untreated mice [23]. Additionally, one of the $B$. microti MAPs (XP_012649271) was tested in mice as a potential vaccine candidate for human babesiosis. Immunization with an E. coli-expressed recombinant form of this MAP induced a Th1 immune response characterized by IgG2a antibody titers and IFN- $\gamma$ production, and provided partial protection against the challenge with $B$. microti [68]. Although the number of boosters and protein amounts needed to achieve this effect would not be practical to apply in humans, these results suggest a potential usefulness of MAPs in future vaccine formulations against B. microti. 


\subsection{Threonine Proteases and the Proteasome}

The proteasome is a cylindrically shaped large complex of proteins in charge of degrading intracellular proteins destined for destruction that have been tagged with polyubiquitin chains, thereby controlling many cellular processes, such as cell cycle progression and cell signaling $[69,70]$. All B. microti threonine proteases are proteasome constituents (seven alpha and seven beta 20S proteasome subunits, Table 1). Additionally, a metalloprotease with a proteasome regulatory function is also listed among the B. microti proteases (XP_021338577 of the M67 family), while assignment of other proteases to this structure needs experimental confirmation. Due to their vital role in cell physiology, drugs targeting proteasome functions have been proposed as therapeutics against several parasitic diseases [71-73]. In the case of Babesia sp., the proteasome inhibitors epoxyketones and boronic acid were shown to reduce the chymotrypsin activity of the proteasome in lysates of $B$. divergens in vitro cultures, leading to the accumulation of poli-ubiquinated proteins and, also, impeding parasite growth in vitro [24]. One of the epoxyketones, carfilzomib, was also assayed in B. microti-infected mice. Carfilzomib is a covalent and irreversible peptide inhibitor of the $\beta 5$ subunit of the human proteasome approved for the clinical treatment of multiple myeloma [74]. Blood lysates of B. microti-infected mice treated with carfilzomib also showed the accumulation of poli-ubiquinated proteins as compared to untreated mice. Moreover, carfilzomib treatment reduced the peak parasitemia levels without apparent toxic effects in the treated mice. Although the dose required to eliminate the parasite would be toxic when applied in humans, these studies indicate that specifically targeting the $B$. microti proteasome would be a possible chemotherapeutic approach against this parasite [24].

\subsection{Comparison between B. microti and B. bovis Functional Proteases}

The genome of $B$. microt $i$ is the smallest among Apicomplexans and encodes $7 \%$ less genes compared to that of $B$. bovis. This difference is mainly due to the large vesa and SmORF multigene families present in B. bovis, which are absent in B. microti [38]. These two gene families encode for highly variable proteins that are involved in escaping the immune system of the vertebrate host and cytoadhesion [75,76]. It remains unknown whether strategies to escape effectors of the immune system exist in B. microti. However, cytoadhesion, especially affecting brain capillaries, has not been described as a major pathogenic mechanism for this parasite [2]. Other unraveled differences include the lack of spherical body proteins in B. microti, consistent with a reduced apical complex [38]. Additionally, contrary to $B$. bovis, B. microti does not have an oligosaccharyl transferase in charge of transferring a (NAcGlc) $)_{2}$ moiety from a lipid-linked oligosaccharide to a nascent protein destined for the secretory pathway in the endoplasmic reticulum. Thus, a significant difference among B. bovis and B. microti is the lack of ability of the latter to produce N-glycosylated proteins [77].

In the present study, we hypothesized that the differences between B. bovis and $B$. microt $i$ include the repertoire of active proteases encoded in their genomes. By orthology searches, we observed that most B. bovis active proteases have an ortholog in B. microti (Supplementary Table S4). The lack of orthology was connected in all but two cases to the expansion of a protease-coding ancestor gene into different numbers of paralogs, which most likely took place after the separation of the most recent common ancestor (MRCA) of B. bovis and B. microti, and thus, they differentiate both species. However, the $\mathrm{S} 8$ family of serine proteases is present with a single member, a subtilisin-like protein (XP_001610126), only in B. bovis but is absent in B. microti. The B. bovis subtilisin-like protein gene is syntenic with orthologous genes in B. divergens, B. ovata, and Babesia sp. Xinjang (data not shown). Importantly, characterization of the subtilisin-like protein of $B$. divergens showed that it localizes to dense granules and contains neutralization-sensitive B-cell epitopes, consistent with a relevant role in the invasion or establishment of the parasite in the infected erythrocyte, as observed for subtilisin-1 and subtilisin-2 in P. falciparum [78-80]. The other case is B. microti SPP aspartic protease (XP_021338622), which is absent in B. 
bovis and other Babesia spp., as mentioned above. The identification of genes absent in B. microti and present in other Babesia spp. or vice versa can allow comprehending the minimum protein dotation needed to fulfill a basic Babesia sp. life cycle, as well as to identify which proteins are associated with species-specific peculiarities and can also be exploited for differential diagnosis, therapeutic, and vaccine developments. B. bovis and B. microti share important similarities in their life cycles, namely being tick-transmitted and having an asexual reproduction stage exclusively within the erythrocytes of their vertebrate hosts. However, they differ in tick and vertebrate host species, as well as by the presence or absence of transovarial transmission in the tick. Transovarial transmission is, indeed, a trademark of the "true" babesias or Babesia sensu stricto, such as B. bovis, while those members of the Babesia genus that do not have this trait, such as B. microti, are considered Babesia sensu lato $[27,28,81]$. These differences are undoubtedly connected with the evolutionary history of B. bovis and B. microti, which can be clearly visualized by their phylogenetic placement into two distant clades (Clades VI and I, respectively, according to Schnittger et al., 2012 and $2021[27,28]$.

\subsection{Non-Peptidase Homologs}

At least 25 non-peptidase homologs are encoded in the B. microti genome (Table 1). A conserved protease domain can be predicted in their sequences, but they lack one or more of the catalytically relevant amino acids. Non-peptidase homologs are commonly found among living organisms and believed to have evolved from catalytically active enzymes. They have lost their catalytic capacity but developed new functions, such as competitive inhibition regulating their active counterparts or even completely new nonprotease-related activities [82,83]. An extreme case of loss of function is observed with a group of paralogs of B. microti that include three metalloproteases of the M41 family and 14 other non-protease members. Different from other non-peptidase homologs, the latter do not have a recognizable protease active site region. According to their conserved domains, their functions include the hydrolysis of nucleoside triphosphates, fusion of vesicles, intracellular transport, and proteasome regulation (Supplementary Table S1).

\subsection{Conclusions and Perspectives}

Proteases are attractive targets against a large number of infectious agents, since many of them are druggable and participate in essential biological processes of pathogenic virus, bacteria, protozoa, and fungi [84]. Indeed, several protease inhibitors are commercially available, and some are successfully employed in the treatment of HIV and Hepatitis $C[85,86]$. The use of protease inhibitors against other relevant viruses, such as dengue and SARS-CoV-2, has also been postulated $[87,88]$.

The present study was aimed at organizing the available information of B. microti proteases and extending the array of identified peptidases encoded in its genome. This information is expected to set the stage for future research directed to understand the biology and pathogenicity of this parasite and to explore proteases as targets for developing novel therapeutic interventions. Recent advances in B. microti gene editing will permit exploring the functional relevance of selected proteases [89,90]. In addition, the application of computer-based inhibitor screening and the use of optimized pipelines to test drug efficacies using in vitro cultures and animal models allows obtaining new therapeutics against human babesiosis in a relatively short period of time [34,91,92]. 


\section{Materials and Methods}

The proteases of B. microti, R1 strain, presented in this study were identified by three different search approaches: (i) extracting and organizing the data available for this parasite in the MEROPS database (www.ebi.ac.uk/merops/, accessed on 1 September 2021) [29], (ii) the identification of homologs of $B$. bovis proteases predicted as active, as reported by Mesplet et al. (2011) [26], and (iii) the search for paralogs of B. microti proteases identified in (i) and (ii). Orthology between B. bovis and B. microti proteases was defined using a BLASTp bidirectional best hit (BBH) approach [93]. Paralogs within the B. microti genome were determined by BLASTp (blast.ncbi.nlm.nih.gov / Blast.cgi, accessed on 1 September 2021), considering a threshold E value of 0.05 . Peptidase domain names and locations were obtained from the Conserved Domains database of the NCBI.

For those proteases included in the MEROPS database and predicted as active, the relevant amino acids of the catalytic site were identified using the data available at this website. For the proteases not included in the MEROPS database, alignments of B. bovis and B. microti orthologs were carried out by Clustal omega [94] (https:/ /www.ebi.ac.uk/Tools/ $\mathrm{msa}$ / clustalo/, accessed on 1 September 2021), and the relevant amino acids described for B. bovis in MEROPS were manually identified for the corresponding B. microti protease. The non-peptidase homologs included those described as such in the MEROPS database. In addition, the peptidases not present in MEROPS were listed as non-peptidase homologs whenever one or more of the catalytically relevant amino acid residues at the homologous positions were missing upon alignment with the sequence of an active proteinase homolog.

The presence of transcripts and translated proteins in the blood parasite stages was evaluated in PiroplasmaDB [95] (piroplasmadb.org/piro/app, accessed on 10.09.2021) and in the proteomic database provided in Reference [31], respectively. The subcellular location of each protease was evaluated by the presence of a signal peptide (SignalP 5.0 server, www.cbs.dtu.dk/services/SignalP/, accessed on 10 September 2021) [96] and transmembrane domains [97] (TMHMM server, www.cbs.dtu.dk/services/TMHMM/, accessed on 10 September 2021) and using the localization predictor DeepLoc-1.0 [98] (www.cbs.dtu.dk/services/DeepLoc/, accessed on 10 September 2021) with the settings for eukaryotic sequences.

Supplementary Materials: The following are available online at https:/ /www.mdpi.com/article/10 .3390 / pathogens10111457/s1, Table S1: M41 metalloprotease paralogue family of B. microti, Table S2: S59 paralogue family of B. microti, Table S3: Predicted subcellular localization of B. microti proteases, Table S4: Comparison between the repertoire of B. microti and B. bovis proteases predicted as active at least in one of either species.

Author Contributions: Conceptualization, M.F.-C. and L.S.; investigation: M.F.-C. and S.N.W.; original draft preparation: M.F.-C.; and writing-reviewing and editing: C.E.S., S.N.W., L.S., and M.F.-C. All authors have read and agreed to the published version of the manuscript.

Funding: We acknowledge the support of projects 2019-PD-E5-I102, 2019-PE-E5-I109, and 2019PE-E5-I105 from the National Institute of Agricultural Technology (INTA, Argentina) and CRIS 2090-32000-039-000-D from ARS-USDA (USA). SNW received a doctoral fellowship from Consejo Nacional de Investigaciones Científicas y Técnicas (CONICET, Argentina).

Institutional Review Board Statement: Not applicable.

Informed Consent Statement: Not applicable.

Data Availability Statement: Not applicable.

Conflicts of Interest: The authors declare no competing interests. 


\section{References}

1. Yang, Y.; Christie, J.; Köster, L.; Du, A.; Yao, C. Emerging Human Babesiosis with “Ground Zero" in North America. Microorganisms 2021, 9, 440. [CrossRef]

2. Vannier, E.; Krause, P.J. Human Babesiosis. N. Engl. J. Med. 2012, 366, 2397-2407. [CrossRef] [PubMed]

3. Tołkacz, K.; Bednarska, M.; Alsarraf, M.; Dwużnik, D.; Grzybek, M.; Welc-Falęciak, R.; Behnke, J.M.; Bajer, A. Prevalence, Genetic Identity and Vertical Transmission of Babesia microti in Three Naturally Infected Species of Vole, Microtus Spp. (Cricetidae). Parasites Vectors 2017, 10, 1-12. [CrossRef]

4. Tufts, D.M.; Diuk-Wasser, M.A. Transplacental Transmission of Tick-Borne Babesia microti in Its Natural Host Peromyscus Leucopus. Parasites Vectors 2018, 11, 1-9. [CrossRef]

5. Iyer, S.; Goodman, K. Congenital Babesiosis From Maternal Exposure: A Case Report. J. Emerg. Med. 2019, 56, e39-e41. [CrossRef]

6. Stanley, J.; Stramer, S.L.; Erickson, Y.; Cruz, J.; Gorlin, J.; Janzen, M.; Rossmann, S.N.; Straus, T.; Albrecht, P.; Pate, L.L.; et al. Detection of Babesia RNA and DNA in Whole Blood Samples from US Blood Donations. Transfusion 2021. [CrossRef]

7. Renard, I.; Ben Mamoun, C. Treatment of Human Babesiosis: Then and Now. Pathogens 2021, 10, 1120. [CrossRef] [PubMed]

8. Krause, P.J.; Auwaerter, P.G.; Bannuru, R.R.; Branda, J.A.; Falck-Ytter, Y.T.; Lantos, P.M.; Lavergne, V.; Meissner, H.C.; Osani, M.C.; Rips, J.G.; et al. Clinical Practice Guidelines by the Infectious Diseases Society of America (IDSA): 2020 Guideline on Diagnosis and Management of Babesiosis. Clin. Infect. Dis. 2021, 72, e49-e64. [CrossRef] [PubMed]

9. Wormser, G.P.; Prasad, A.; Neuhaus, E.; Joshi, S.; Nowakowski, J.; Nelson, J.; Mittleman, A.; Aguero-Rosenfeld, M.; Topal, J.; Krause, P.J. Emergence of Resistance to Azithromycin-Atovaquone in Immunocompromised Patients with Babesia microti Infection. Clin. Infect. Dis. 2010, 50, 381-386. [CrossRef] [PubMed]

10. Simon, M.S.; Westblade, L.F.; Dziedziech, A.; Visone, J.E.; Furman, R.R.; Jenkins, S.G.; Schuetz, A.N.; Kirkman, L.A. Clinical and Molecular Evidence of Atovaquone and Azithromycin Resistance in Relapsed Babesia microti Infection Associated With Rituximab and Chronic Lymphocytic Leukemia. Clin. Infect. Dis. 2017, 65, 1222-1225. [CrossRef] [PubMed]

11. Florin-Christensen, M.; Schnittger, L. Piroplasmids and Ticks: A Long-Lasting Intimate Relationship. Front. Biosci. 2009, 14, 3064-3073. [CrossRef] [PubMed]

12. Antunes, S.; Rosa, C.; Couto, J.; Ferrolho, J.; Domingos, A. Deciphering Babesia-Vector Interactions. Front. Cell. Infect. Microbiol. 2017, 7, 429. [CrossRef] [PubMed]

13. Klemba, M.; Goldberg, D.E. Biological Roles of Proteases in Parasitic Protozoa. Annu. Rev. Biochem. 2003, 71, 275-305. [CrossRef] [PubMed]

14. Cai, H.; Kuang, R.; Gu, J.; Wang, Y. Proteases in Malaria Parasites-A Phylogenomic Perspective. Curr. Genom. 2011, 12, 417-427. [CrossRef] [PubMed]

15. Lilburn, T.G.; Cai, H.; Zhou, Z.; Wang, Y. Protease-Associated Cellular Networks in Malaria Parasite Plasmodium falciparum. BMC Genom. 2011, 12, 1-16. [CrossRef]

16. Rosenthal, P.; Sijwali, P.; Singh, A.; Shenai, B. Cysteine Proteases of Malaria Parasites: Targets for Chemotherapy. Curr. Pharma. Des. 2002, 8, 1659-1672. [CrossRef]

17. Li, H.; Child, M.A.; Bogyo, M. Proteases as Regulators of Pathogenesis: Examples from the Apicomplexa. Biochim. Biophys. Acta (BBA)-Proteins Proteom. 2012, 1824, 177-185. [CrossRef]

18. Pandey, K.C. Centenary Celebrations Article: Cysteine proteases of human malaria parasites. J. Parasit. Dis. 2011, 35, 94-103. [CrossRef]

19. Sedwick, C. Plasmepsin V, a Secret Weapon Against Malaria. PLoS Biol. 2014, 12, e1001898. [CrossRef]

20. Zhang, N.-Z.; Xu, Y.; Wang, M.; Petersen, E.; Chen, J.; Huang, S.-Y.; Zhu, X.-Q. Protective Efficacy of Two Novel DNA Vaccines Expressing Toxoplasma Gondii Rhomboid 4 and Rhomboid 5 Proteins against Acute and Chronic Toxoplasmosis in Mice. Expert Rev. Vaccines 2015, 14, 1289-1297. [CrossRef]

21. Okubo, K.; Yokoyama, N.; Govind, Y.; Alhassan, A.; Igarashi, I. Babesia bovis: Effects of Cysteine Protease Inhibitors on in vitro Growth. Exp. Parasitol. 2007, 117, 214-217. [CrossRef] [PubMed]

22. AbouLaila, M.; Nakamura, K.; Govind, Y.; Yokoyama, N.; Igarashi, I. Evaluation of the in Vitro Growth-Inhibitory Effect of Epoxomicin on Babesia Parasites. Vet. Parasitol. 2010, 167, 19-27. [CrossRef] [PubMed]

23. Munkhjargal, T.; Ishizaki, T.; Guswanto, A.; Takemae, H.; Yokoyama, N.; Igarashi, I. Molecular and Biochemical Characterization of Methionine Aminopeptidase of Babesia bovis as a Potent Drug Target. Vet. Parasitol. 2016, 221, 14-23. [CrossRef]

24. Jalovecka, M.; Hartmann, D.; Miyamoto, Y.; Eckmann, L.; Hajdusek, O.; O’Donoghue, A.J.; Sojka, D. Validation of Babesia Proteasome as a Drug Target. Int. J. Parasitol. Drugs Drug Res. 2018, 8, 394-402. [CrossRef] [PubMed]

25. Schwake, C.; Baldwin, M.R.; Bachovchin, W.; Hegde, S.; Schiemer, J.; Okure, C.; Levin, A.E.; Vannier, E.; Hanada, T.; Chishti, A.H. HIV Protease Inhibitors Block Parasite Signal Peptide Peptidases and Prevent Growth of Babesia microti Parasites in Erythrocytes. Biochem. Biophys. Res. Commun. 2019, 517, 125-131. [CrossRef]

26. Mesplet, M.; Palmer, G.H.; Pedroni, M.J.; Echaide, I.; Florin-Christensen, M.; Schnittger, L.; Lau, A.O.T. Genome-Wide Analysis of Peptidase Content and Expression in a Virulent and Attenuated Babesia bovis Strain Pair. Mol. Biochem. Parasitol. 2011, 179, 111-113. [CrossRef] [PubMed]

27. Schnittger, L.; Rodriguez, A.E.; Florin-Christensen, M.; Morrison, D.A. Babesia: A World Emerging. Infect. Genet. Evol. 2012, 12, 1788-1809. [CrossRef] 
28. Schnittger, L.; Ganzinelli, S.; Bhoora, R.; Omondi, D.; Nijhof, A.M.; Florin-Christensen, M. The Piroplasmida Babesia, Cytauxzoon, and Theileria in Farm and Companion Animals: Species Compilation, Molecular Phylogeny, and Evolutionary Insights. Parasitol. Res. 2021. under revision.

29. Rawlings, N.D.; Bateman, A. How to Use the MEROPS Database and Website to Help Understand Peptidase Specificity. Protein Sci. 2021, 30, 83-92. [CrossRef]

30. Silva, J.C.; Cornillot, E.; McCracken, C.; Usmani-Brown, S.; Dwivedi, A.; Ifeonu, O.O.; Crabtree, J.; Gotia, H.T.; Virji, A.Z.; Reynes, C.; et al. Genome-Wide Diversity and Gene Expression Profiling of Babesia microti Isolates Identify Polymorphic Genes That Mediate Host-Pathogen Interactions. Sci. Rep. 2016, 6, 1-15. [CrossRef]

31. Magni, R.; Luchini, A.; Liotta, L.; Molestina, R.E. Analysis of the Babesia microti proteome in infected red blood cells by a combination of nanotechnology and mass spectrometry. Int. J. Parasitol. 2019, 49, 139-144. [CrossRef]

32. Florin-Christensen, M.; Florin-Christensen, J.; Tiedtke, A.; Rasmussen, L. New aspects of extracellular hydrolytic enzymes in lower eukaryotes. Eur. J. Cell Biol. 1989, 48, 1-4.

33. Binder, E.M.; Kim, K. Location, location, location: Trafficking and function of secreted proteases of Toxoplasma and Plasmodium. Traffic 2004, 5, 914-924. [CrossRef]

34. Šnebergerová, P.; Bartošová-Sojková, P.; Jalovecká, M.; Sojka, D. Plasmepsin-like Aspartyl Proteases in Babesia. Pathogens 2021, 10, 1241. [CrossRef] [PubMed]

35. Li, X.; Chen, H.; Bahamontes-Rosa, N.; Kun, J.F.J.; Traore, B.; Crompton, P.D.; Chishti, A.H. Plasmodium falciparum Signal Peptide Peptidase Is a Promising Drug Target against Blood Stage Malaria. Biochem. Biophys. Res. Commun. 2009, 380, 454-459. [CrossRef] [PubMed]

36. Gluzman, I.Y.; Francis, S.E.; Oksman, A.; Smith, C.E.; Duffin, K.L.; Goldberg, D.E. Order and Specificity of the Plasmodium falciparum Hemoglobin Degradation Pathway. J. Clin. Investig. 1994, 93, 1602-1608. [CrossRef]

37. Nasamu, A.S.; Polino, A.J.; Istvan, E.S.; Goldberg, D.E. Malaria Parasite Plasmepsins: More than Just Plain Old Degradative Pepsins. J. Biolog. Chem. 2020, 295, 8425-8441. [CrossRef] [PubMed]

38. Cornillot, E.; Hadj-Kaddour, K.; Dassouli, A.; Noel, B.; Ranwez, V.; Vacherie, B.; Augagneur, Y.; Brès, V.; Duclos, A.; Randazzo, S.; et al. Sequencing of the Smallest Apicomplexan Genome from the Human Pathogen Babesia Microti. Nucleic Acids Res. 2012, 40, 9102-9114. [CrossRef]

39. Kim, K. Role of Proteases in Host Cell Invasion by Toxoplasma gondii and Other Apicomplexa. Acta Tropica 2004, 91, 69-81. [CrossRef]

40. Cowman, A.F.; Crabb, B.S. Invasion of Red Blood Cells by Malaria Parasites. Cell 2006, 124, 755-766. [CrossRef]

41. Ettari, R.; Previti, S.; di Chio, C.; Zappalà, M. Falcipain-2 and Falcipain-3 Inhibitors as Promising Antimalarial Agents. Curr. Med. Chem. 2021, 28, 3010-3031. [CrossRef]

42. Hanspal, M.; Dua, M.; Takakuwa, Y.; Chishti, A.H.; Mizuno, A. Plasmodium falciparum Cysteine Protease Falcipain-2 Cleaves Erythrocyte Membrane Skeletal Proteins at Late Stages of Parasite DevelopmentPresented in Part in Abstract Form at the 43rd Annual Meeting of the American Society of Hematology, Orlando, FL, 2001. Blood 2002, 100, 1048-1054. [CrossRef]

43. Sijwali, P.S.; Rosenthal, P.J. Gene Disruption Confirms a Critical Role for the Cysteine Protease Falcipain-2 in Hemoglobin Hydrolysis by Plasmodium falciparum. Proc. Natl. Acad. Sci. USA 2004, 101, 4384-4389. [CrossRef]

44. Mesplet, M.; Echaide, I.; Dominguez, M.; Mosqueda, J.J.; Suarez, C.E.; Schnittger, L.; Florin-Christensen, M. Bovipain-2, the falcipain-2 ortholog, is expressed in intraerythrocytic stages of the tick-transmitted hemoparasite Babesia bovis. Parasites Vectors 2010, 3, 113. [CrossRef] [PubMed]

45. Martins, T.M.; Gonçalves, L.M.D.; Capela, R.; Moreira, R.; do Rosário, V.E.; Domingos, A. Effect of Synthesized Inhibitors on Babesipain-1, a New Cysteine Protease from the Bovine Piroplasm Babesia bigemina. Transbound. Emerg. Dis. 2010, 57, 68-69. [CrossRef] [PubMed]

46. Martins, T.M.; do Rosário, V.E.; Domingos, A. Expression and Characterization of the Babesia bigemina Cysteine Protease BbiCPL1. Acta Tropica 2012, 121, 1-5. [CrossRef]

47. Carletti, T.; Barreto, C.; Mesplet, M.; Mira, A.; Weir, W.; Shiels, B.; Oliva, A.G.; Schnittger, L.; Florin-Christensen, M. Characterization of a Papain-like Cysteine Protease Essential for the Survival of Babesia ovis Merozoites. Ticks Tick-Borne Dis. 2016, 7, 85-93. [CrossRef] [PubMed]

48. Lu, S.; Ascencio, M.E.; Torquato, R.J.S.; Florin-Christensen, M.; Tanaka, A.S. Kinetic Characterization of a Novel Cysteine Peptidase from the Protozoan Babesia bovis, a Potential Target for Drug Design. Biochimie 2020, 179, 127-134. [CrossRef]

49. Wei, N.; Du, Y.; Lu, J.; Zhou, Y.; Cao, J.; Zhang, H.; Gong, H.; Zhou, J. A Cysteine Protease of Babesia microti and Its Interaction with Tick Cystatins. Parasitol. Res. 2020, 119, 3013-3022. [CrossRef]

50. Wu, J.; Cao, J.; Zhou, Y.; Zhang, H.; Gong, H.; Zhou, J. Evaluation on Infectivity of Babesia microti to Domestic Animals and Ticks Outside the Ixodes Genus. Front. Microbiol. 2017, 8, 1915. [CrossRef]

51. Lu, S.; da Rocha, L.A.; Torquato, R.J.S.; da Silva Vaz Junior, I.; Florin-Christensen, M.; Tanaka, A.S. A Novel Type 1 Cystatin Involved in the Regulation of Rhipicephalus microplus Midgut Cysteine Proteases. Ticks Tick-Borne Dis. 2020, 11, 101374. [CrossRef]

52. Ascencio, M.E.; Florin-Christensen, M.; Mamoun, C.B.; Weir, W.; Shiels, B.; Schnittger, L. Cysteine Proteinase C1A Paralog Profiles Correspond with Phylogenetic Lineages of Pathogenic Piroplasmids. Vet. Sci. 2018, 5, 41. [CrossRef] [PubMed]

53. Dogga, S.K.; Soldati-Favre, D. Biology of Rhomboid Proteases in Infectious Diseases. Semin. Cell Dev. Biol. 2016, 60, 38-45. [CrossRef] [PubMed] 
54. Düsterhöft, S.; Künzel, U.; Freeman, M. Rhomboid Proteases in Human Disease: Mechanisms and Future Prospects. Biochim. Et Biophys. Acta (BBA)-Mol. Cell Res. 2017, 1864, 2200-2209. [CrossRef] [PubMed]

55. Dowse, T.J.; Soldati, D. Rhomboid-like Proteins in Apicomplexa: Phylogeny and Nomenclature. Trends Parasitol. 2005, 21, 254-258. [CrossRef] [PubMed]

56. Santos, J.M.; Graindorge, A.; Soldati-Favre, D. New Insights into Parasite Rhomboid Proteases. Mol. Biochem. Parasitol. 2012, 182, 27-36. [CrossRef] [PubMed]

57. Lin, J.; Meireles, P.; Prudêncio, M.; Engelmann, S.; Annoura, T.; Sajid, M.; Chevalley-Maurel, S.; Ramesar, J.; Nahar, C.; Avramut, C.M.C.; et al. Loss-of-Function Analyses Defines Vital and Redundant Functions of the Plasmodium Rhomboid Protease Family. Mol. Microbiol. 2013, 88, 318-338. [CrossRef]

58. Urban, S. Making the Cut: Central Roles of Intramembrane Proteolysis in Pathogenic Microorganisms. Nat. Rev. Microbiol. 2009, 7, 411-423. [CrossRef]

59. Gandhi, S.; Baker, R.P.; Cho, S.; Stanchev, S.; Strisovsky, K.; Urban, S. Designed Parasite-Selective Rhomboid Inhibitors Block Invasion and Clear Blood-Stage Malaria. Cell Chem. Biol. 2020, 27, 1410-1424. [CrossRef]

60. Han, Y.; Zhou, A.; Lu, G.; Zhao, G.; Wang, L.; Guo, J.; Song, P.; Zhou, J.; Zhou, H.; Cong, H.; et al. Protection via a ROM4 DNA Vaccine and Peptide against Toxoplasma gondii in BALB/c Mice. BMC Infect. Dis. 2017, 17, 1-9. [CrossRef]

61. Gallenti, R.; Poklepovich, T.; Florin-Christensen, M.; Schnittger, L. The Repertoire of Serine Rhomboid Proteases of Piroplasmids of Importance to Animal and Human Health. Int. J. Parasitol. 2021, 51, 455-462. [CrossRef]

62. Lysyk, L.; Brassard, R.; Touret, N.; Lemieux, M.J. PARL Protease: A Glimpse at Intramembrane Proteolysis in the Inner Mitochondrial Membrane. J. Mol. Biol. 2020, 432, 5052-5062. [CrossRef] [PubMed]

63. Freeman, M. Proteolysis within the Membrane: Rhomboids Revealed. Nat. Rev. Mol. Cell Biol. 2004, 5, 188-197. [CrossRef] [PubMed]

64. Ueti, M.W.; Johnson, W.C.; Kappmeyer, L.S.; Herndon, D.R.; Mousel, M.R.; Reif, K.E.; Taus, N.S.; Ifeonu, O.O.; Silva, J.C.; Suarez, C.E.; et al. Transcriptome Dataset of Babesia bovis Life Stages within Vertebrate and Invertebrate Hosts. Data Brief 2020, $33,106533$. [CrossRef] [PubMed]

65. Wright, I.G.; Goodger, B.V.; Mahoney, D.F. Virulent and Avirulent Strains of Babesia bovis: The Relationship Between Parasite Protease Content and Pathophysiological Effect of the Strain. J. Protozool. 1981, 28, 118-120. [CrossRef]

66. Lowther, W.T.; Matthews, B.W. Structure and Function of the Methionine Aminopeptidases. Biochim. Et Biophys. Acta (BBA)-Protein Struct. Mol. Enzymol. 2000, 1477, 157-167. [CrossRef]

67. Naughton, J.A.; Nasizadeh, S.; Bell, A. Downstream Effects of Haemoglobinase Inhibition in Plasmodium falciparum-Infected Erythrocytes. Mol. Biochem. Parasitol. 2010, 173, 81-87. [CrossRef]

68. Munkhjargal, T.; Yokoyama, N.; Igarashi, I. Recombinant Methionine Aminopeptidase Protein of Babesia microti: Immunobiochemical Characterization as a Vaccine Candidate against Human Babesiosis. Parasitol. Res. 2016, 115, 3669-3676. [CrossRef] [PubMed]

69. Ciechanover, A. The Ubiquitin-Proteasome Proteolytic Pathway. Cell 1994, 79, 13-21. [CrossRef]

70. Bedford, L.; Paine, S.; Sheppard, P.W.; Mayer, R.J.; Roelofs, J. Assembly, Structure, and Function of the 26S Proteasome. Trends Cell Biol. 2010, 20, 391-401. [CrossRef]

71. Li, H.; Bogyo, M.; Fonseca, P.C.A. da The Cryo-EM Structure of the Plasmodium falciparum 20S Proteasome and Its Use in the Fight against Malaria. FEBS J. 2016, 283, 4238-4243. [CrossRef] [PubMed]

72. Bijlmakers, M.J. Ubiquitination and the Proteasome as Drug Targets in Trypanosomatid Diseases. Front. Chem. 2021, 8. [CrossRef] [PubMed]

73. Thomas, M.; Brand, S.; de Rycker, M.; Zuccotto, F.; Lukac, I.; Dodd, P.G.; Ko, E.-J.; Manthri, S.; McGonagle, K.; Osuna-Cabello, M.; et al. Scaffold-Hopping Strategy on a Series of Proteasome Inhibitors Led to a Preclinical Candidate for the Treatment of Visceral Leishmaniasis. J. Med. Chem. 2021, 64, 5905-5930. [CrossRef] [PubMed]

74. Kuhn, D.J.; Chen, Q.; Voorhees, P.M.; Strader, J.S.; Shenk, K.D.; Sun, C.M.; Demo, S.D.; Bennett, M.K.; van Leeuwen, F.W.B.; Chanan-Khan, A.A.; et al. Potent Activity of Carfilzomib, a Novel, Irreversible Inhibitor of the Ubiquitin-Proteasome Pathway, against Preclinical Models of Multiple Myeloma. Blood 2007, 110, 3281-3290. [CrossRef]

75. Allred, D.R.; Carlton, J.M.R.; Satcher, R.L.; Long, J.A.; Brown, W.C.; Patterson, P.E.; O'Connor, R.M.; Stroup, S.E. The Ves Multigene Family of B. bovis Encodes Components of Rapid Antigenic Variation at the Infected Erythrocyte Surface. Mol. Cell 2000, 5, 153-162. [CrossRef]

76. Ferreri, L.M.; Brayton, K.A.; Sondgeroth, K.S.; Lau, A.O.T.; Suarez, C.E.; McElwain, T.F. Expression and Strain Variation of the Novel "Small Open Reading Frame" (Smorf) Multigene Family in Babesia bovis. Int. J. Parasitol. 2012, 42, 131-138. [CrossRef]

77. Florin-Christensen, M.; Rodriguez, A.E.; Suárez, C.E.; Ueti, M.W.; Delgado, F.O.; Echaide, I.; Schnittger, L. N-Glycosylation in Piroplasmids: Diversity within Simplicity. Pathogens 2021, 10, 50. [CrossRef]

78. Montero, E.; Gonzalez, L.M.; Rodriguez, M.; Oksov, Y.; Blackman, M.J.; Lobo, C.A. A Conserved Subtilisin Protease Identified in Babesia divergens Merozoites. J. Biol. Chem. 2006, 281, 35717-35726. [CrossRef]

79. Harris, P.K.; Yeoh, S.; Dluzewski, A.R.; O’Donnell, R.A.; Withers-Martinez, C.; Hackett, F.; Bannister, L.H.; Mitchell, G.H.; Blackman, M.J. Molecular Identification of a Malaria Merozoite Surface Sheddase. PLoS Pathogens 2005, 1, e29. [CrossRef] 
80. Koussis, K.; Withers-Martinez, C.; Yeoh, S.; Child, M.; Hackett, F.; Knuepfer, E.; Juliano, L.; Woehlbier, U.; Bujard, H.; Blackman, M.J. A Multifunctional Serine Protease Primes the Malaria Parasite for Red Blood Cell Invasion. EMBO J. 2009, 28, 725-735. [CrossRef]

81. Jalovecka, M.; Sojka, D.; Ascencio, M.; Schnittger, L. Babesia Life Cycle-When Phylogeny Meets Biology. Trends Parasitol. 2019, 35, 356-368. [CrossRef] [PubMed]

82. Todd, A.E.; Orengo, C.A.; Thornton, J.M. Sequence and Structural Differences between Enzyme and Nonenzyme Homologs. Structure 2002, 10, 1435-1451. [CrossRef]

83. Reynolds, S.L.; Fischer, K. Pseudoproteases: Mechanisms and Function. Biochem. J. 2015, 468, 17-24. [CrossRef]

84. Agbowuro, A.A.; Huston, W.M.; Gamble, A.B.; Tyndall, J.D.A. Proteases and protease inhibitors in infectious diseases. Med. Care Res. Rev. 2018, 38, 1295-1331. [CrossRef]

85. Fernández-Montero, J.V.; Barreiro, P.; Soriano, V. HIV protease inhibitors: Recent clinical trials and recommendations on use. Expert Opin. Pharm. 2009, 10, 1615-1629. [CrossRef]

86. Rowe, I.A.; Mutimer, D.J. Protease inhibitors for treatment of genotype 1 hepatitis C virus infection. The BMJ 2011,343 , d6972. [CrossRef]

87. Mushtaq, M.; Naz, S.; Parang, K.; Ul-Haq, Z. Exploiting Dengue Virus Protease as a Therapeutic Target; Current Status, Challenges and Future Avenues. Curr. Med. Chem. 2021, in press. [CrossRef] [PubMed]

88. Srivastava, K.; Singh, M.K. Drug repurposing in COVID-19: A review with past, present and future. Metab. Open 2021, $12,100121$. [CrossRef]

89. Jaijyan, D.K.; Govindasamy, K.; Singh, J.; Bhattacharya, S.; Singh, A.P. Establishment of a stable transfection method in Babesia microti and identification of a novel bidirectional promoter of Babesia microti. Sci. Rep. 2020, 10, 15614. [CrossRef]

90. Liu, M.; Ji, S.; Rizk, M.A.; Adjou Moumouni, P.F.; Galon, E.M.; Li, J.; Li, Y.; Zheng, W.; Benedicto, B.; Tumwebaze, M.A.; et al. Transient Transfection of the Zoonotic Parasite Babesia microti. Pathogens 2020, 9, 108. [CrossRef]

91. Pérez, B.; Antunes, S.; Gonçalves, L.M.; Domingos, A.; Gomes, J.R.; Gomes, P.; Teixeira, C. Toward the discovery of inhibitors of babesipain-1, a Babesia bigemina cysteine protease: In vitro evaluation, homology modeling and molecular docking studies. $J$. Comput. -Aided Mol. Des. 2013, 27, 823-835. [CrossRef]

92. Yin, M.; Zhang, H.B.; Tao, Y.; Yao, J.M.; Liu, H.; Win, H.H.; Huo, L.L.; Jiang, B.; Chen, J.X. Optimization of an Evaluation Method for Anti-Babesia microti Drug Efficacy. Acta Tropica 2021, 225, 106179. [CrossRef]

93. Tatusov, R.L.; Koonin, E.V.; Lipman, D.J. A Genomic Perspective on Protein Families. Science 1997, 278, 631-637. [CrossRef] [PubMed]

94. Sievers, F.; Higgins, D.G. Clustal Omega for Making Accurate Alignments of Many Protein Sequences. Protein Sci. 2018, 27, 135-145. [CrossRef] [PubMed]

95. Aurrecoechea, C.; Barreto, A.; Brestelli, J.; Brunk, B.P.; Cade, S.; Doherty, R.; Fischer, S.; Gajria, B.; Gao, X.; Gingle, A.; et al. EuPathDB: The Eukaryotic Pathogen Database. Nucleic Acids Res. 2013, 41, D684-D691. [CrossRef]

96. Almagro Armenteros, J.J.; Tsirigos, K.D.; Sønderby, C.K.; Petersen, T.N.; Winther, O.; Brunak, S.; von Heijne, G.; Nielsen, H. SignalP 5.0 Improves Signal Peptide Predictions Using Deep Neural Networks. Nat. Biotechnol. 2019, 37, 420-423. [CrossRef]

97. Krogh, A.; Larsson, B.; von Heijne, G.; Sonnhammer, E.L.L. Predicting Transmembrane Protein Topology with a Hidden Markov Model: Application to Complete Genomes. J. Mol. Biol. 2001. [CrossRef]

98. Almagro Armenteros, J.J.; Sønderby, C.K.; Sønderby, S.K.; Nielsen, H.; Winther, O. DeepLoc: Prediction of Protein Subcellular Localization Using Deep Learning. Bioinformatics 2017, 33, 3387-3395. [CrossRef] 